\title{
Probing quantum spin liquids in equilibrium using the inverse spin Hall effect
}

\author{
Joshua Aftergood $\odot$ and So Takei \\ Department of Physics, Queens College of the City University of New York, Queens, New York 11367, USA \\ and Physics Doctoral Program, The Graduate Center of the City University of New York, New York, New York 10016, USA
}

(Received 9 March 2020; revised 3 July 2020; accepted 27 August 2020; published 18 September 2020)

\begin{abstract}
We propose an experimental method utilizing a strongly spin-orbit coupled metal to quantum magnet bilayer that will probe quantum magnets lacking long-range magnetic order, e.g., quantum spin liquids, via examination of the voltage noise spectrum in the metal layer. The bilayer is held in thermal and chemical equilibrium, and spin fluctuations arising across the single interface are converted into voltage fluctuations in the metal as a result of the inverse spin Hall effect. We elucidate the theoretical workings of the proposed bilayer system and provide precise predictions for the frequency characteristics of the enhancement to the ac electrical resistance measured in the metal layer for three candidate quantum spin liquid models. Application to the Heisenberg spin-1/2 kagomé lattice model should allow for the extraction of any spinon gap present. A quantum spin liquid consisting of fermionic spinons coupled to a U(1) gauge field should cause subdominant $\Omega^{4 / 3}$ scaling of the resistance of the coupled metal. Finally, if the magnet is well-captured by the Kitaev model in the gapless spin liquid phase, then the proposed bilayer can extract the two-flux gap which arises in spite of the gapless spectrum of the fermions. We, therefore, show that spectral analysis of the ac resistance in the metal in a single interface, equilibrium bilayer can test the relevance of a quantum spin liquid model to a given candidate material.
\end{abstract}

DOI: 10.1103/PhysRevResearch.2.033439

\section{INTRODUCTION}

Quantum spin liquids (QSLs) refer to intriguing states of quantum spin systems in which strong quantum fluctuations prevent spins from ordering down to zero temperature and the prototypical wave function exhibits extensive many-body entanglement $[1,2]$. They are endowed with fascinating physical properties like nonlocal excitations $[3,4]$ and nontrivial topology [5,6], and substantial pioneering work has been accomplished in the pursuit of a physical instantiation of this long-sought-after phase of matter. The most promising candidates to date include the mineral herbertsmithite [7], certain organic salt compounds [8], and the so-called Kitaev materials [9], and their experimental studies have ranged from nuclear magnetic resonance [10-13], to susceptibility and heat capacity investigations [14-16], to thermal transport studies [17-22], and to neutron scattering [23-26]. Theoretical works have shown that QSL ground states are realized in the antiferromagnetic $S=1 / 2$ Heisenberg model on the kagomé lattice [27-31], in models that couple low-energy fermionic spin excitations to an emergent U(1) gauge field [32-37], as well as in the exactly solvable Kitaev model [38] in two $[39,40]$ and three $[41,42]$ dimensions. However, while the number and types of potential QSL models have proliferated, the experimental methods available for studying them have

Published by the American Physical Society under the terms of the Creative Commons Attribution 4.0 International license. Further distribution of this work must maintain attribution to the author(s) and the published article's title, journal citation, and DOI. not [43]. It is therefore important to the search for compounds possessing QSL ground states to propose previously unknown techniques by which to probe and categorize candidate materials.

The inverse spin Hall effect (ISHE) [44] is an essential component of the spintronics repertoire [45] and provides an opportunity to develop probes of unconventional quantum magnets. This phenomenon has found utility in detecting spin currents induced by thermal gradients [46-48] and in performing nonlocal spin transport experiments through magnetic insulators [49-53]. The prime function of the ISHE is that it acts as a transducer between spin and charge current densities in metals with strong spin-orbit coupling. One may therefore envisage a bilayer system in which a strongly spin-orbit coupled heavy-element metal (e.g., Pt, Ta, W) is deposited on top of a quantum magnet, i.e., consider coupling the metal to a spin dissipating subsystem that freezes out charge degrees of freedom to focus solely on the spin sector, while holding the entire bilayer in thermal and chemical equilibrium. The addition of a spin dissipating subsystem will result in equilibrium spin current fluctuations across the interface [54,55], which, as a result of the ISHE, are converted into charge fluctuations inside the metal-charge fluctuations that may encode information about the microscopic structure of the quantum magnet. The QSL-to-normal-metal bilayer therefore presents a table-top setup that can be used to electrically probe the low-energy density of states of the QSL material. This type of bilayer system has garnered some study with respect to ferromagnetic insulators hosting magnons [56]; however, the utility of the ISHE as a noise conversion mechanism has not yet been explored in the context of exotic quantum magnets in general and the search for QSLs in particular. 
In this work, we propose a relatively simple mechanism that can probe exotic $2 \mathrm{~d}$ QSL ground states possessing gauge fluctuations and extensive many-body entanglement via equilibrium or near-equilibrium measurements. We will develop the theory underpinning the aforementioned QSL-tonormal-metal bilayer that utilizes the QSL material as a spin dissipating subsystem, where spin dissipation via the QSL material results in spin noise generation due to the fluctuation dissipation theorem. The normal metal layer acts as a resistive element, and in the quantum limit, i.e., at very low temperatures, the power spectrum of the asymmetrized spin noise in the metal is entirely quantum in nature [57] and encodes information about the spin sector of the QSL layer. We show that the asymmetrized spin noise generated in this manner must affect, e.g., the ac resistance measured in the normal metal, if the normal metal possesses strong spin-orbit coupling. We then use our theory to examine three QSL models: the $S=1 / 2$ nearest-neighbor Heisenberg antiferromagnet on the kagomé lattice, a model consisting of gapless fermionic spin excitations coupled to an emergent $\mathrm{U}(1)$ gauge field, and the bare Kitaev honeycomb model in the gapless spin liquid phase.

We consider a gapped ground state in our approach to the kagomé lattice antiferromagnet owing to DMRG studies that indicate the presence of a gap [29], in addition to gapped out flux excitations, and utilize a bosonic parton mean-field theory [27] when characterizing the emergent low-energy spin excitations. In so doing, we show that our proposed bilayer system can successfully quantify any spinon gap present.

Motivated by the fact that the slave-rotor representation [58] of the Hubbard model on a triangular lattice results in stable QSL mean-field states comprised of a spinon Fermi surface coupled to a $\mathrm{U}(1)$ gauge field [33] that may apply to existing candidate materials [e.g., $\kappa$-(BEDT-TTF $)_{2}-\mathrm{Cu}_{2}(\mathrm{CN})_{3}$ and $\left.\mathrm{YbMgGaO}_{4}\right]$, we examine this QSL model theoretically in our proposed bilayer system. We find that a QSL model coupling gapless fermions with a Fermi surface to an emergent $U(1)$ gauge field produces a subdominant $\Omega^{4 / 3}$ frequency correction to the ac resistance measured across the normal metal that our proposed system can educe.

Finally, our treatment of the bare, gapless Kitaev model considers the isotropic point of the quantum spin liquid phase, where all bond interactions are equal in strength, and assumes a flux-free background-a valid assumption for temperatures lower than $1 \%$ of the bond strength $[59,60]$. Under these considerations we find that when coupled to a normal metal, the presence of a Kitaev QSL will allow characterization of the two-flux gap energy that emerges in spite of the gapless nature of the fermion spectrum.

\section{THEORY}

Let us consider coupling an insulating spin system (i.e., a quantum magnet) to a strongly spin-orbit coupled heavyelement metal, as depicted in Fig. 1. The addition of the spin system gives rise to increased spin dissipation, which, as a result of the fluctuation-dissipation theorem (FDT), contributes additional spin fluctuations inside the metal. If strong spin-orbit interactions are present in the conductor (as in, e.g., $\mathrm{Pt}, \mathrm{Ta}, \mathrm{W}[61,62])$, then FDT requires that spin fluctuations

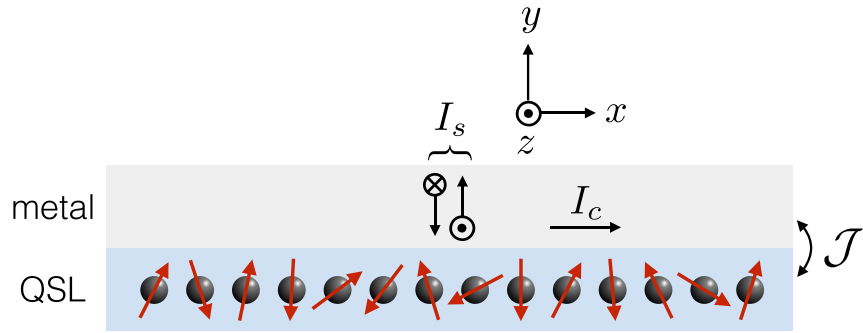

FIG. 1. A cartoon of the proposed system: a quantum spin liquid to normal metal bilayer in equilibrium, where fluctuating spin current $I_{s}$ across the interface becomes fluctuating charge current $I_{c}$ (or fluctuating voltage) in the normal metal via the inverse spin Hall effect and results in measurable modifications to the ac resistance of the metal.

across the interface will give rise to voltage fluctuations in the conductor via the ISHE, i.e., $S=S_{0}+\delta S$, where $S$ is the total voltage noise in the metal in thermal equilibrium, $S_{0}$ is the total noise present in the bare metal, and $\delta S$ is the portion of the noise that arises due to the presence of the quantum magnet.

In the quantum limit-when the sample is cooled to as low a temperature as possible-background thermal noise in the metal is strongly suppressed, and

$$
S(\Omega)=4 \hbar \Omega R(\Omega) \theta(\Omega)
$$

Here, the Heaviside step function $\theta(\Omega)$ signifies that only positive frequencies are possible in the quantum limit. This indicates that the metal is able to absorb quasiparticles from an external system, e.g., a detector, but is unable to emit them [57]. The voltage fluctuations present in the metal are thus entirely due to the presence of the QSL candidate material and any background quantum noise.

We assume that in the quantum limit the base resistance is essentially constant in the frequency range of interest and known in a given strongly spin-orbit coupled metal, and therefore any quantum background noise linear in frequency that arises can be accounted for and removed, exposing $\delta S(\Omega)$.

In the upcoming sections, we provide the technical calculations necessary to extract the correction to the equilibrium voltage noise $\delta S(\Omega)$ - the enhancement to the ac power spectrum of the voltage noise in a strongly spin-orbit coupled metal interfaced with a general quantum magnet possessing no long-ranged order-and then apply our results to some well-known QSL models. Characteristic features in the frequency distribution will then allow for discriminating between the QSL ground states of the various candidate materials via a relatively straightforward near-equilibrium measurements of the ac resistance present in the metal layer via Eq. (1).

We consider a bilayer system as shown in Fig. 1, comprised of a thin normal metal layer (thickness $d$ ) affixed atop a QSL material. The interface is set at the $x z$ plane, and the QSL is treated as a $2 \mathrm{~d}$ lattice of fixed quantum spins $\boldsymbol{S}_{\boldsymbol{i}}$. The particular lattice structures of the QSLs come into play later in the discussion. We assume that the entire heterostructure is thermalized to a temperature $T$ in order to eliminate the effects of nonequilibrium drives. 
We assume the QSL and the normal metal are coupled via exchange interaction

$$
H_{c}=-\mathcal{J} v_{0} \sum_{i} \boldsymbol{s}\left(y=0, \boldsymbol{r}_{i}\right) \cdot \boldsymbol{S}_{\boldsymbol{i}},
$$

where $v_{0}$ is the volume in the metal per spin of the QSL, $\mathcal{J}$ is the exchange constant, and $\boldsymbol{r}_{\boldsymbol{i}}$ is a vector of the interfacial coordinates at the $y=0$ plane specifying the position of $S_{i}$. The local spin density of the metal is given by $s(x)=$ $(1 / 2) \psi_{s}^{\dagger}(\boldsymbol{x}) \boldsymbol{\tau}_{s s^{\prime}} \psi_{s^{\prime}}(\boldsymbol{x})$, where $\boldsymbol{\tau}$ is the vector of Pauli matrices and $s$ labels the electron spin quantum number.

The interfacial spin current operator $I_{s}$ is defined by considering the total $z$ polarized spin entering the metal, i.e.,

$$
\begin{aligned}
I_{s}(t)= & (-\iota) \frac{\mathcal{J} v_{0}}{2} \sum_{i}\left[s^{-}\left(y=0, \boldsymbol{r}_{i}, t\right) S_{i}^{+}(t)\right. \\
& \left.-s^{+}\left(y=0, \boldsymbol{r}_{i}, t\right) S_{i}^{-}(t)\right],
\end{aligned}
$$

where $\iota=\sqrt{-1}$. The spin current noise across the interface can then be computed to lowest non-trivial order in $\mathcal{J}$ using, e.g., the real-time Keldysh formalism and the result formally takes the form [63]

$$
\begin{aligned}
\left\langle I_{s}(t) I_{s}(0)\right\rangle= & \left(\frac{\mathcal{J} v_{0}}{2}\right)^{2} \sum_{i j}\left\langle s^{-}\left(y=0, \boldsymbol{r}_{i}, t\right) s^{+}\left(y=0, \boldsymbol{r}_{j}, 0\right)\right\rangle \\
& \times\left[\left\langle S_{i}^{+}(t) S_{j}^{-}(0)\right\rangle+\left\langle S_{i}^{-}(t) S_{j}^{+}(0)\right\rangle\right],
\end{aligned}
$$

where $\langle\cdots\rangle$ represents correlation functions with respect to the unperturbed Hamiltonian. The spectrum of the interfacial spin current noise is then defined via

$$
S_{s}(\Omega)=\int_{-\infty}^{\infty} d t\left\langle I_{s}(t) I_{s}(0)\right\rangle e^{i \Omega t} .
$$

The spin correlation function in the metallic sector can be readily computed, so we obtain a general expression for the equilibrium spin current noise spectrum at finite temperature [63],

$$
\begin{aligned}
S_{S}(\Omega, T)= & 2 \iota\left(\frac{\mathcal{J} v_{0} m k_{F}}{2 \pi^{2} \hbar}\right)^{2} \sum_{i j} \int_{-\infty}^{\infty} d v \frac{v-\Omega}{e^{\beta \hbar(v-\Omega)}-1} \\
& \times \operatorname{sinc}^{2}\left(k_{F}\left|\boldsymbol{r}_{\boldsymbol{i}}-\boldsymbol{r}_{\boldsymbol{j}}\right|\right)\left[\chi_{i j}^{+-}(v)+\chi_{i j}^{-+}(v)\right],
\end{aligned}
$$

where $k_{F}$ is the Fermi wave vector of the metal, and we have introduced the dynamical spin correlation function of the QSL,

$$
\chi_{i j}^{\mp \pm}(v) \equiv-\iota \int d t\left\langle S_{i}^{\mp}(t) S_{j}^{ \pm}(0)\right\rangle e^{i v t},
$$

to account for the portion of the noise that arises due to spin fluctuations in the QSL. Finally, for large Fermi wave vectors, i.e., $k_{F}\left|\boldsymbol{r}_{\boldsymbol{i}}-\boldsymbol{r}_{\boldsymbol{j}}\right| \gg 1$ for all $\boldsymbol{i}, \boldsymbol{j}$, Eq. (6) can be approximated in spatially local terms, and we obtain

$$
\begin{aligned}
S_{S}(\Omega, T) \approx & 2 \iota\left(\frac{\mathcal{J} v_{0} m k_{F}}{2 \pi^{2} \hbar}\right)^{2} \sum_{i} \int_{-\infty}^{\infty} d v \frac{v-\Omega}{e^{\beta \hbar(\nu-\Omega)}-1} \\
& \times\left[\chi_{i i}^{+-}(v)+\chi_{i i}^{-+}(v)\right] .
\end{aligned}
$$

We emphasize here that the derivation has so far assumed no particular form for the QSL Hamiltonian. Instead, Eq. (8) is constructed in order to apply to noise generated in a strongly spin-orbit coupled normal metal due to the proximity of a general quantum magnet (albeit with no long-ranged magnetic order), such that extracting a usable quantity depends only on characterizing $\chi_{i i}^{ \pm \mp}(v)$ for a given QSL model.

The ISHE finally converts the $z$ polarized spin current density in the $y$ direction into a charge current density along the $x$ direction (see Fig. 1). The conversion ratio between the interfacial spin current fluctuations and the measurable voltage fluctuations may depend nontrivially on various properties of the metal, e.g., its spin Hall angle, geometry, and spin diffusion length. However, as shown in, e.g., Refs. [63,64], these details may be lumped into a single phenomenological spin-to-voltage noise conversion constant $\Theta$ so that the ac voltage fluctuations generated by the quantum magnet can be expressed in terms of the spin fluctuations in Eq. (8) as

$$
\delta S(\Omega, T)=\Theta S_{S}(\Omega, T) .
$$

Therefore, what we have calculated in Eq. (8) is directly related to the ac voltage noise in the metal generated by the presence of the quantum magnet. We may then extract the modification to the resistance acquired by the presence of the quantum magnet from this quantity using Eq. (1).

\section{APPLICATION TO QUANTUM SPIN LIQUIDS}

We now consider three types of QSL models in the context of our proposed bilayer system: the antiferromagnetic Heisenberg $S=1 / 2$ kagomé lattice model (HKLM); a model involving a spinon Fermi surface coupled to an emergent U(1) gauge field; and the Kitaev honeycomb model in the gapless spin liquid phase. We ultimately show that the bilayer system we propose will allow the extraction of characteristic frequency dependencies in each case. All three models above are believed to be of relevance for the descriptions of some QSL candidate materials. Detailed discussion of these connections will be presented in Sec. V.

\section{A. Heisenberg antiferromagnet on the kagomé lattice}

The HKLM is an important prototypical model that supports a QSL ground state, and is believed to be a relevant model for the mineral compound $\mathrm{ZnCu}_{3}(\mathrm{OH})_{6} \mathrm{Cl}_{2}$ (herbertsmithite), one of the prime contenders for an experimental realization of a QSL. Recent neutron scattering experiments suggest that the relevant theoretical model for the QSL state observed in this compound consists of the so-called $Z_{2}$ spin liquids $[27,65,66]$. These QSL states possess two types of excitations, i.e., the $S=1 / 2$ spinon and a gapped vortex, also known as a vison, corresponding to an emergent $Z_{2}$ gauge field [5]. It was shown recently that these visons can act as a momentum sink for the spinons and can flatten the dynamic spin structure factor [66] in accordance with experimental observations of herbertsmithite. However, for the purposes of the heterostructure, we are proposing in this work, we note that only local spin fluctuations are important and visons do not themselves carry spin. Therefore we expect that visons should not significantly renormalize spin current fluctuations, and that the reshuffling of the spin quasiparticle spectral weight in momentum space should not drastically affect local quantities. 


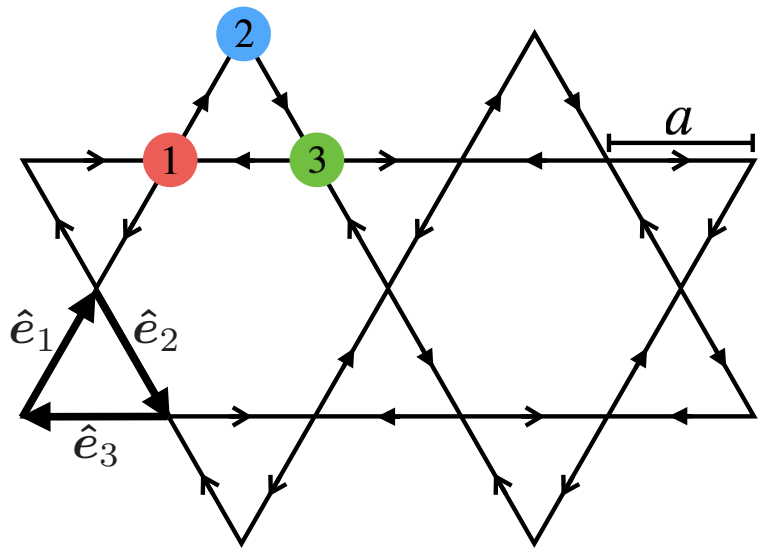

FIG. 2. The kagomé lattice, with positions on a representative unit cell marked. The $\hat{\boldsymbol{e}}_{i}$ represent the unit vectors of a unit cell, and $a$ is the length of a bond. Arrows between sites correspond to the direction dependent $Q_{i j}$. Solid arrows around upward facing triangles depict $Q_{i j}=Q_{1}$, and wire arrows around downward facing triangles depict $Q_{i j}=Q_{2}$.

We therefore ignore the effects of the visons in this subsection, follow the calculations performed in Ref. [27] for the HKLM, and apply the result to the observable we are proposing as a probe of the QSL state.

We begin with the Hamiltonian for the bare HKLM

$$
H_{\mathrm{HKLM}}=-\frac{J}{2} \sum_{\langle i, j\rangle} S_{i} \cdot S_{j},
$$

where $\langle\boldsymbol{i}, \boldsymbol{j}\rangle$ indicates nearest-neighbor pairings and $J$ is the exchange coupling. Mean-field approaches to characterizing the HKLM ground states include bosonic representations [27] of the gapped spinons and fermionic representations, where a gap arises due to pairing in the Hamiltonian [67]. In what follows, we focus on the bosonic representation of the QSL phase as done in Ref. [27].

We begin with the standard Schwinger boson representation of the spin operators $\boldsymbol{S}_{\boldsymbol{i}}=(1 / 2) b_{\boldsymbol{i} \sigma}^{\dagger} \boldsymbol{\tau}_{\sigma \sigma^{\prime}} b_{\boldsymbol{i} \sigma^{\prime}}$, where $b_{i \sigma}$ represents a bosonic spinon, and then use the mean-field decoupling $Q_{i j}=(1 / 2)\left\langle\varepsilon_{\sigma \sigma^{\prime}} b_{i \sigma} b_{j \sigma^{\prime}}\right\rangle$, where $\varepsilon_{\sigma \sigma^{\prime}}$ is the completely antisymmetric tensor of SU(2) and the field satisfies $Q_{i j}=-Q_{j i}$. Substituting this representation into Eq. (10), the mean-field decoupling results in the Hamiltonian

$$
H_{\mathrm{HKLM}}=-\frac{J}{2} \sum_{\langle i, \boldsymbol{j}\rangle} \sum_{\sigma \sigma^{\prime}} Q_{i j} \varepsilon_{\sigma \sigma^{\prime}} b_{\boldsymbol{i} \sigma}^{\dagger} b_{\boldsymbol{j} \sigma^{\prime}}^{\dagger}+\text { H.c. }+\lambda \sum_{\boldsymbol{i}, \sigma} b_{\boldsymbol{i} \sigma}^{\dagger} b_{\boldsymbol{i} \sigma},
$$

where H.c. denotes the hermitian conjugate and $\lambda$ is a Lagrange multiplier that constrains the model to one boson per site.

Figure 2 shows a depiction of the kagomé lattice, where the arrows between sites in a unit cell correspond to the direction dependent $Q_{i j}$, i.e., $Q_{1}$ and $Q_{2}$ are the two distinct expectation values of $Q_{i j}$. In the figure, solid arrows correspond to $Q_{i j}=$ $Q_{1}$ and wire arrows correspond to $Q_{i j}=Q_{2}$. The two possible locally stable QSL mean-field solutions [27,65,66,68] occur when $Q_{1}=Q_{2}=Q$, corresponding to $\pi$ flux through the

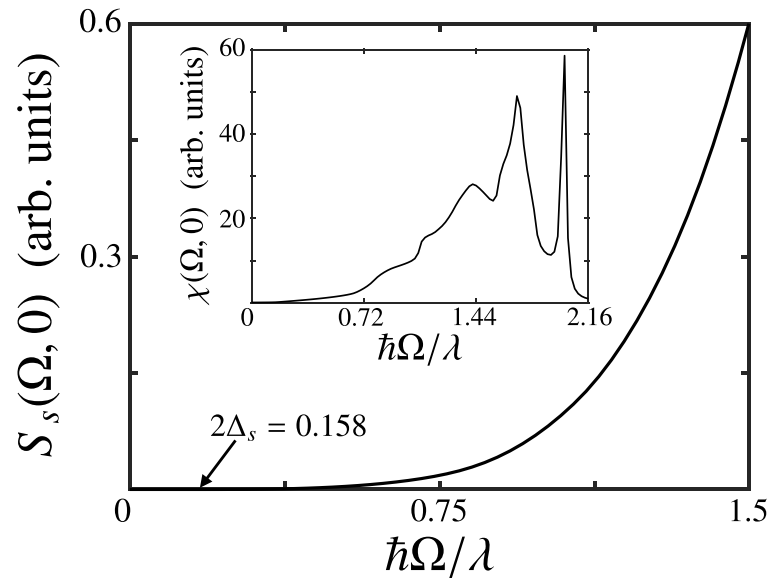

FIG. 3. A numerical plot showing $S_{s}(\Omega, 0)$ in the HKLM as a function of ac frequency normalized by the mean-field coupling, $\lambda=0.695 J$. The main plot shows the computed sum. The graph is identically zero until a critical frequency of twice the gap energy is achieved, $\hbar \Omega_{c}=2 \Delta_{s}=0.158 \lambda$, at which point it becomes possible to create spinon pairs in the QSL and noise enhancement commences. The inset is the ac susceptibility $\chi(\Omega, 0)$ in the case of the HKLM, which can be extracted by numerical differentiation of Eq. (8), and is in good agreement with previous works, see, e.g., Ref. [69].

hexagonal plaquette, and $Q_{1}=-Q_{2}$, corresponding to no flux through the plaquette, both with $Q_{1}, Q_{2} \in \mathbb{R}$. Here, we will specifically consider the $\pi$ flux case and utilize mean-field theoretical values put forward in Ref. [66] for our purposes.

Upon diagonalizing Eq. (11), we can solve for the total spin susceptibility $\chi(v)=\sum_{i}\left[\chi_{i i}^{+-}(v)+\chi_{i i}^{-+}(v)\right]$, and use it to derive the noise correction. We perform the finite temperature calculation in Appendix A, and then take the $T \rightarrow 0$ limit. Our resultant expression for the zero temperature ac noise power spectrum then becomes

$$
\begin{aligned}
S_{S}(\Omega, 0)= & \frac{4 \pi}{N_{u} \hbar}\left(\frac{\mathcal{J} v_{0} m k_{F}}{2 \pi^{2} \hbar}\right)^{2} \sum_{\boldsymbol{k}, \boldsymbol{q}} \sum_{n, m, l} U_{n m}^{\boldsymbol{k} *} U_{n m}^{\boldsymbol{k}} U_{\bar{n} l}^{\boldsymbol{q} *} U_{\bar{n} l}^{\boldsymbol{q}} \\
& \times\left(\hbar \Omega-\epsilon_{\boldsymbol{k} m}-\epsilon_{\boldsymbol{q} l}\right) \theta\left(\hbar \Omega-\epsilon_{\boldsymbol{k} m}-\epsilon_{\boldsymbol{q} l}\right),
\end{aligned}
$$

where $U_{n m}^{k}$ is the $6 \times 6$ matrix that diagonalizes Eq. (11), $\epsilon_{k m}$ is the $m$ th energy eigenvalue of the diagonalized Hamiltonian, and $\theta(x)$ is the Heaviside theta function. Note that $\bar{n}=n+3$, the index $n=1,2,3$, and $m, l=1, \ldots, 6$. We now analyze this expression numerically.

Figure 3 is a plot of Eq. (12), from which we find that, in the quantum limit, it is possible to extract an estimate of the spin gap using our proposed bilayer. We use the variable values proffered in Refs. [66,69] for the HKLM, taking as a reasonable estimate for the spin gap $\Delta_{s}=0.055 \mathrm{~J}$. In the HKLM, the gap energy can be found by setting $\boldsymbol{k}=0$ and finding the minimal eigenvalue [68], with the outcome $\Delta_{s}=\sqrt{\lambda^{2}-12 J^{2} Q^{2}}$. Therefore, rather than computing the values of $\lambda$ and $Q$ variationally, we set $\lambda=0.695 \mathrm{~J}$ and tune $Q$ such that $\Delta_{s}=0.055 \mathrm{~J}$, resulting in $Q=0.2$. Note that $Q$ quantifies antiferromagnetic correlations and is restricted to $|Q| \leqslant 1 / \sqrt{2}$, at which point nearest-neighbor spins form 
singlets and the model experiences a phase transition into an ordered phase.

Importantly, the HKLM models a highly frustrated antiferromagnetic material, and therefore includes quantum fluctuations. At very low temperatures, quantum fluctuations are required in order to drive the interfacial equilibrium noise generated as a result of the mechanism we are considering. Figure 3 shows that no noise enhancement is expected until probing frequencies greater than the critical frequency $\hbar \Omega_{c}=2 \Delta_{s}$, at which point production of a pair of spinons can occur in the QSL and an enhancement in the noise begins to manifest. Therefore probing this region of the frequency range for a given material that is adequately modeled by the HKLM should allow for a direct estimate of the spin gap, $\Delta_{s}$, present in that material.

\section{B. Spinon Fermi surface coupled to an emergent U(1) gauge field}

Some QSL candidate materials, e.g., organic salt compounds [32-34] and $\mathrm{YbMgGaO}_{4}$ [70-72], may be described as spinon Fermi seas, where the spin susceptibility is strongly renormalized by an emergent $U(1)$ gauge field. In this section, we first compute (using the Keldysh functional integral formalism) the thermal spin current fluctuations arising in the metal due to the presence of fermions in the QSL material, and second how the gauge field renormalization affects those thermal fluctuations in our proposed bilayer system at finite temperature. We then take the $T \rightarrow 0$ limit at the end and present the zero temperature ac frequency result.

Let us begin with the real-time action for $\mathcal{N}$ flavors of fermionic spinons coupled to a compact U(1) gauge field in $2+1$ dimensions $[33,73,74]$

$$
\begin{aligned}
S= & \sum_{\sigma} \int d t d \boldsymbol{x}\left\{\bar{c}_{\sigma}(t, \boldsymbol{x})\left(\iota \hbar \partial_{t}+\mu\right) c_{\sigma}(t, \boldsymbol{x})\right. \\
& \left.-\frac{1}{2 m_{s}} \bar{c}_{\sigma}(t, \boldsymbol{x})[-\iota \hbar \nabla+\boldsymbol{a}(t, \boldsymbol{x})]^{2} c_{\sigma}(t, \boldsymbol{x})\right\},
\end{aligned}
$$

where $c_{\sigma}(t, \boldsymbol{x})$ is a spinon Grassmann field, $\sigma$ is the flavor in$\operatorname{dex}, m_{s}$ is the spinon effective mass, $\mu$ is the spinon chemical potential, and $\boldsymbol{a}(t, \boldsymbol{x})$ is the gauge field. The (real-frequency) retarded RPA propagator for the gauge fluctuations in the Coulomb gauge can be obtained by analytically continuing the standard result from the imaginary-time formalism $[73,75]$

$$
\begin{aligned}
D_{\xi \xi^{\prime}}^{R}(\boldsymbol{q}, \Omega) & =-\left(\delta_{\xi \xi^{\prime}}-\frac{q_{\xi} q_{\xi^{\prime}}}{q^{2}}\right) \frac{1}{\mathcal{N}} \frac{1}{\chi_{d q}^{2}-\iota \frac{E_{F s}}{\pi \hbar^{3}} \frac{\Omega}{v_{F s}} q}, \\
& \equiv-\left(\delta_{\xi \xi^{\prime}}-\frac{q_{\xi} q_{\xi^{\prime}}}{q^{2}}\right) d_{\boldsymbol{q}}^{R}(\Omega),
\end{aligned}
$$

where $\xi, \xi^{\prime}$ label the Cartesian components, $v_{F s}=\hbar k_{F s} / m_{s}$ is the spinon Fermi velocity, $k_{F s}$ is the spinon Fermi wave vector, $E_{F s}$ is the spinon Fermi energy, and $\chi_{d}=\left(24 \hbar m_{s}\right)^{-1}$ is the Landau diamagnetic susceptibility of the fermions.

Lastly, we define the interaction portion of the Keldysh action by placing Eq. (13) on the Keldysh contour and extracting the term dependent on the gauge field to first order,

$$
\begin{aligned}
S_{\text {int }}= & -\frac{1}{2 \hbar \sqrt{\mathscr{A}}} \int_{-\infty}^{\infty} d t \sum_{\boldsymbol{k} \boldsymbol{k}^{\prime} \sigma} \sum_{\xi=x, z} \sum_{\eta= \pm} \eta \\
& \times v_{\boldsymbol{k}+\boldsymbol{k}^{\prime} s}^{\xi} \bar{c}_{\boldsymbol{k} \sigma}\left(t^{\eta}\right) a_{\boldsymbol{k}-\boldsymbol{k}^{\prime}}^{\xi}\left(t^{\eta}\right) c_{\boldsymbol{k}^{\prime} \sigma}\left(t^{\eta}\right),
\end{aligned}
$$

where $\eta= \pm$ represents the Keldysh time-loop forward and backward branches, $v_{\boldsymbol{k} s}=\hbar \boldsymbol{k} / m_{s}$, and $\mathscr{A}$ is the total area of the QSL.

\section{Noise correction due to bare susceptibility}

The spin operators of the QSL are represented using Abrikosov fermions, i.e., $\boldsymbol{S}_{\boldsymbol{i} \sigma}=(1 / 2) c_{i \sigma}^{\dagger} \boldsymbol{\tau}_{\sigma \sigma^{\prime}} c_{\boldsymbol{i \sigma ^ { \prime }}}$, where the constraint of one fermion per site is enforced. Starting from the calculation of the bare bubble, without gauge field renormalization, we can find the uncorrected noise enhancement generated across the interface by first calculating the total susceptibility, $\chi^{(0)}(v)=\sum_{i}\left[\chi_{i i}^{+-(0)}(v)+\chi_{i i}^{-+(0)}(v)\right]$, given by

$$
\begin{aligned}
\chi^{(0)}(v)= & -2 \iota \sum_{i} \int d t e^{\imath v t} \\
& \times\left\langle T_{K} \bar{c}_{i \downarrow}\left(t^{-}\right) c_{i \uparrow}\left(t^{-}\right) \bar{c}_{i \uparrow}\left(0^{+}\right) c_{i \downarrow}\left(0^{+}\right)\right\rangle,
\end{aligned}
$$

where $T_{K}$ is the Keldysh time ordering operator. Equation (16) quantifies the portion of the susceptibility that arises due simply to the presence of the fermions in the QSL material, without the additional corrections from emergent gauge photons, and evaluates to

$$
\chi^{(0)}(v)=-\iota N \frac{a_{s}^{2} m_{s}^{2}}{\pi \hbar^{2}} \frac{v}{1-e^{-\beta \hbar v}},
$$

where $N$ is the total number of lattice points and $a_{s}$ is the area occupied by each QSL spin. By inserting Eq. (17) into Eq. (8) and then moving to the zero temperature limit the zeroth order ac noise correction is therefore found to be

$$
S_{s}^{(0)}(\Omega, 0)=\frac{N}{3 \pi}\left(\frac{\mathcal{J} v_{0} m k_{F}}{2 \pi^{2} \hbar}\right)^{2}\left(\frac{m_{s} a_{s}}{\hbar}\right)^{2} \Omega^{3} .
$$

\section{Gauge field correction to the noise}

The next nonzero correction to the susceptibility arises due to gauge field renormalization and is given by the secondorder expansion using Eq. (15) as the perturbation,

$$
\begin{aligned}
\chi_{i i}^{\mp \pm(2)}(v)= & \frac{\iota}{2} \int d t e^{i v t} \\
& \times\left\langle T_{K} \bar{c}_{i \downarrow \uparrow}\left(t^{-}\right) c_{i \uparrow \downarrow}\left(t^{-}\right) \bar{c}_{i \uparrow \downarrow}\left(0^{+}\right) c_{i \downarrow \uparrow}\left(0^{+}\right) S_{\mathrm{int}}^{2}\right\rangle .
\end{aligned}
$$

Expanding this gives five possible diagrams, only three of which contribute and must therefore be considered; these three are diagrammatically depicted in Fig. 4. It is important when calculating gauge invariant quantities to sum over all three contributing diagrams, because only then do the divergences cancel exactly [75,76].

Accounting for the required diagrams (see Appendix B for details of the calculation), the total susceptibility at two-loop 


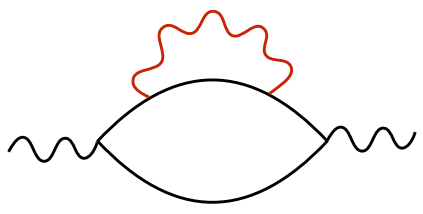

(a)

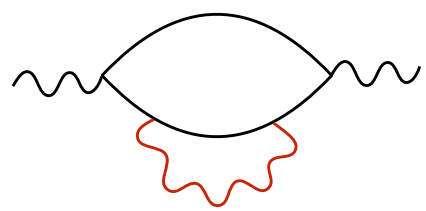

(b)

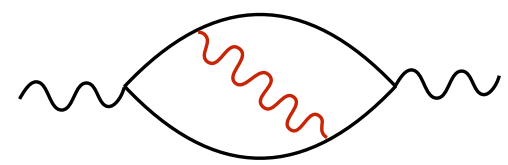

(c)

FIG. 4. Three diagrams contributing to the $\mathcal{O}(1 / \mathcal{N})$ correction to the spin susceptibility generated by the presence of $U(1)$ gauge field fluctuations.

order $\chi^{(2)}(v)=\sum_{i}\left[\chi_{i i}^{+-(2)}(v)+\chi_{i i}^{-+(2)}(v)\right]$ reads

$$
\begin{aligned}
\chi^{(2)}(\nu)= & \frac{N}{1-e^{-\beta \hbar \nu}} \frac{m_{s}^{2} a_{s}^{2}}{2(2 \pi)^{2} \hbar^{4}} \int \frac{d \omega}{2 \pi} \int_{0}^{\infty} d z F(\nu, \omega) \\
& \times\left[d_{-q}^{R}(-\omega)-d_{-q}^{A}(-\omega)\right]\left(1-z^{-1} \tan ^{-1} z\right),
\end{aligned}
$$

where

$$
\begin{aligned}
F(v, \omega)= & \int \frac{d \omega^{\prime}}{2 \pi}\left[\tanh \left(\frac{\hbar\left(\omega^{\prime}+v\right)}{2 k_{B} T}\right)-\tanh \left(\frac{\hbar \omega^{\prime}}{2 k_{B} T}\right)\right] \\
& \times\left[2 \operatorname{coth}\left(\frac{\hbar \omega}{2 k_{B} T}\right)-\tanh \left(\frac{\hbar\left(\omega^{\prime}+\omega+v\right)}{2 k_{B} T}\right)\right. \\
& \left.-\tanh \left(\frac{\hbar\left(\omega^{\prime}+\omega\right)}{2 k_{B} T}\right)\right] .
\end{aligned}
$$

We can then express the second order ac voltage noise correction as

$$
\begin{aligned}
\delta S_{s}^{(2)}(\Omega, T)= & \iota N\left(\frac{\mathcal{J} v_{0} m k_{F}}{2 \pi^{2} \hbar}\right)^{2} \frac{m_{s}^{2} a_{s}^{2}}{(2 \pi)^{2} \hbar^{4}} \\
& \times \int d v \frac{v-\Omega}{e^{\beta \hbar(\nu-\Omega)}-1} \int \frac{d \omega}{2 \pi} \int_{0}^{\infty} d z \frac{F(\nu, \omega)}{1-e^{-\beta \hbar \nu}} \\
& \times\left[d_{-q}^{R}(-\omega)-d_{-q}^{A}(-\omega)\right]\left(1-z^{-1} \tan ^{-1} z\right) .
\end{aligned}
$$

We now move into the zero temperature limit, which allows completion of each of the integrals. We expand the $q$ integral to lowest order in $\omega$ for $\omega \ll \Omega_{F s}$, where $\Omega_{F s}$ is the spinon Fermi frequency. Thus the ac noise correction due to gauge fluctuations finally becomes

$$
\delta S_{s}^{(2)}(\Omega, 0)=\frac{0.075}{\mathcal{N}}\left(\frac{\Omega}{\Omega_{F s}}\right)^{4 / 3} S_{s}^{(0)}(\Omega, 0) .
$$

We can therefore see from the zeroth and second order calculations that the total ac voltage fluctuations generated across the normal metal sample when interfaced with a QSL material possessing an emergent $\mathrm{U}(1)$ gauge field will have a $\Omega^{3}$ lowest order temperature dependence modified by a $\Omega^{4 / 3}$ subdominant component. The total ac noise correction generated is

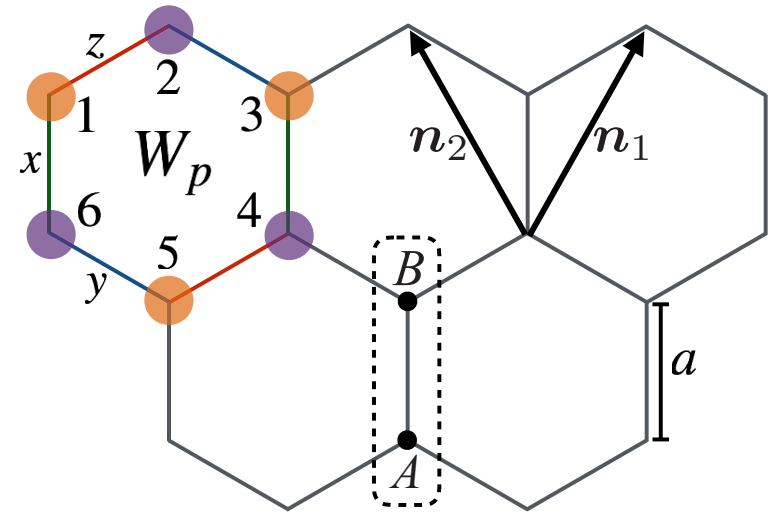

FIG. 5. The Kitaev honeycomb lattice, where the two sublattices are shown by the purple (sublattice $A$ ) and orange (sublattice $B$ ) dots. Representative $x, y$, and $z$ links are depicted. The flux operator on plaquette $p, W_{p}$, is shown, $\boldsymbol{n}_{1}$ and $\boldsymbol{n}_{2}$ are the primitive lattice vectors, and $a$ is the lattice spacing. Also depicted is a representative unit cell enclosed in the dashed box.

given by the sum of the two terms, such that the power spectrum of the enhancement becomes

$$
S_{s}(\Omega, 0)=S_{s}^{(0)}(\Omega, 0)\left[1+\frac{0.075}{\mathcal{N}}\left(\frac{\Omega}{\Omega_{F s}}\right)^{4 / 3}\right],
$$

when considering the regime $\Omega \ll \Omega_{F s}$.

That a noise enhancement accrues due to the presence of an emergent gauge field is physically understandable as the collective fermionic spin excitations present, i.e., the spinons, acquiring a higher scattering probability due to the added presence of gauge fluctuations. Additional scattering must result in additional noise generation. This enhancement should be extricable in the quantum limit using Eq. (1): first plotting $\delta R(\Omega) / \Omega^{2}$ and then subtracting off the intercept reveals the bare $\Omega^{4 / 3}$ correction term characteristic of the gauge field renormalization calculated in Eq. (24).

\section{The Kitaev honeycomb model}

The second example of a $Z_{2}$ QSL is the Kitaev model on the honeycomb lattice (see Fig. 5), where exchange frustration arising due to the inability to simultaneously satisfy all Kitaev interactions along neighboring bonds can drive the system into a QSL phase. The Kitaev spin liquid is exactly solvable, and we select this example for consideration in our proposed system for that reason, in addition to the fact that there are potential material candidates available currently $[13,26]$. Furthermore, we restrict our investigation to the gapless phase, where the Kitaev exchange couplings are equal. However, while fermionic excitations are gapless, the emergent $Z_{2}$ gauge field is not, and this has the peculiar effect of generating an apparent gap in the fermionic sector [40,77]. The Kitaev model therefore provides something of a synthesis of the last two sections, as it is both a $Z_{2}$ and gapless theory. We give a short overview of the approach to solving the Kitaev model following Refs. [38,78], and then apply the solution to extracting an observable out of our proposed heterostructure. 
The Kitaev model on the honeycomb lattice is given by [38]

$$
H_{K}=\sum_{\gamma,\langle i, j\rangle_{\gamma}}-K_{\gamma} S_{i}^{\gamma} S_{j}^{\gamma}
$$

where $\gamma=\{x, y, z\}$ represents the different nearest-neighbor bond directions (see Fig. 5) at each lattice point with interaction strength $K_{\gamma}$. In understanding the gapless spin liquid phase of the Kitaev model, parton mean-field theories have been proposed $[38,79]$ that characterize the emergent excitations as Dirac fermions [78] arising in tandem with an emergent gapped flux.

This can be seen by first representing the spin operators in terms of four Majorana fermions, i.e., $S_{i}^{\gamma}=\imath f_{i \gamma} c_{i}$ with $\left\{f_{i \gamma}, f_{i^{\prime} \gamma^{\prime}}\right\}=2 \delta_{i i^{\prime}} \delta_{\gamma \gamma^{\prime}},\left\{c_{i}, c_{i^{\prime}}\right\}=2 \delta_{i i^{\prime}}$, and $\left\{f_{i \gamma}, c_{i^{\prime}}\right\}=0$, which then gives $S_{i}^{\gamma} S_{j}^{\gamma}=-\imath \hat{u}_{i j} c_{i} c_{j}$, where $\hat{u}_{i j}=\iota \sum_{\gamma} f_{i \gamma} f_{j \gamma}$ is the bond operator. Noting that the bond operators commute with each other and with any bilinear operator containing $c_{i}$, it is possible to replace them with their eigenvalues \pm 1 . One thus obtains $S_{i}^{\gamma} S_{j}^{\gamma}= \pm \iota c_{i} c_{j}$, and $H_{K}$ becomes bilinear in the Majorana fermions. Second, we note that the so-called flux operator on a plaquette $W_{p}=f_{1 x} f_{2 y} f_{3 z} f_{4 x} f_{5 y} f_{6 z}$, where the subscript $p$ labels the plaquette number, commutes with the Hamiltonian and is therefore an integral of motion. When a plaquette has an even number of bonds, as Fig. 5 makes clear is the case for the honeycomb lattice, its eigenvalues are \pm 1 . Finally, we note that the spin representation in terms of four Majorana fermions enlarges the Hilbert space from two to four, and must therefore be constrained in order to recover the physical Hilbert space. This constraint is enforced via a projection operator $P_{i}=(1 / 2)\left(1+f_{i x} f_{i y} f_{i z} c_{i}\right)$ for each site $\boldsymbol{i}$, which requires that the initial spin algebra be conserved. Effectively, what has been done is to reduce the initial Hamiltonian to a noninteracting Dirac fermion hopping Hamiltonian in a static $Z_{2}$ gauge field, where the choice of values for $\hat{u}_{i j}$ amounts to fixing a gauge, and the gauge invariant quantities are the plaquette operators $W_{p}$.

A theorem by Lieb [80] guarantees that if the number of sites per plaquette is $2 \bmod 4$, then the ground state is in the flux-free sector, i.e., where it is possible to set $\hat{u}_{i j}=1$. The ground state is therefore described by the free Majorana hopping Hamiltonian,

$$
H_{K}=\iota K \sum_{\langle i, j\rangle} c_{i} c_{j}
$$

where $K_{x}=K_{y}=K_{z} \equiv K$, and the subscripts $\boldsymbol{i}$ and $\boldsymbol{j}$ are now labeling $A$ and $B$ sites respectively.

Equation (26) can be expressed in terms of complex fermions using a standard procedure [40]. We take $\boldsymbol{r}$ as a unit cell coordinate, comprised of an $A$ site and a $B$ site as shown in Fig. 5, and combine the two Majorana species into a single complex fermion species through $b_{r}=\left(c_{A r}+\iota c_{B r}\right) / 2$. Putting the system on a torus with $N_{u}$ unit cells, and changing to reciprocal space with $b_{r}=N_{u}^{-1 / 2} \sum_{k} e^{l k r} b_{k}$, Eq. (26) becomes

$$
H_{K}=\sum_{k}\left(\begin{array}{ll}
b_{k}^{\dagger} & b_{-k}
\end{array}\right)\left(\begin{array}{cc}
\varepsilon_{k} & \iota \Delta_{k} \\
-\iota \Delta_{k} & -\varepsilon_{k}
\end{array}\right)\left(\begin{array}{c}
b_{k} \\
b_{-k}^{\dagger}
\end{array}\right)
$$

where $\varepsilon_{k}=\operatorname{Re}\left(s_{k}\right), \Delta_{k}=\operatorname{Im}\left(s_{k}\right), s_{k}=K\left(1+e^{\imath \boldsymbol{k} n_{1}}+e^{\imath \boldsymbol{k} \boldsymbol{n}_{2}}\right)$, and the primitive vectors $\boldsymbol{n}_{1}$ and $\boldsymbol{n}_{2}$ are defined in Fig. 5. Thus the ground state energy is $E_{0}=-\sum_{k}\left|s_{k}\right|[38,40]$.

The isotropic Kitaev spin liquid offers a unique opportunity in that only on-site and nearest-neighbor spin correlations contribute [81]. Therefore we use Eq. (6) in calculating the noise and include both on-site and nearest-neighbor contributions under the assumption that the envelope $\operatorname{sinc}^{2}\left(k_{F}\left|\boldsymbol{r}_{i}-\boldsymbol{r}_{j}\right|\right)$ does not noticeably vary over the lattice constant $a$. We then find that the total susceptibility $\chi(v)=\sum_{i j}\left[\chi_{i j}^{+-}(v)+\right.$ $\left.\chi_{i j}^{-+}(v)\right]$ becomes

$$
\begin{aligned}
\chi(\nu)= & -8 \iota N_{u} \int d t\left(\left\langle S_{A 0}^{x}(t) S_{A 0}^{x}(0)\right\rangle\right. \\
& \left.+\left\langle S_{A 0}^{x}(t) S_{B 0}^{x}(0)\right\rangle\right) e^{\imath v t},
\end{aligned}
$$

where we invoke the translation invariance of the isotropic spin liquid to select the $\boldsymbol{r}=0$ unit cell.

The above two dynamical spin correlation functions can be written as [81]

$$
\begin{aligned}
& \left\langle S_{A 0}^{x}(t) S_{A 0}^{x}(0)\right\rangle=\left\langle e^{\iota H_{K} t / \hbar} c_{A 0} e^{-\iota\left(H_{K}+V_{x}\right) t / \hbar} c_{A 0}\right\rangle_{K} \\
& \left\langle S_{A 0}^{x}(t) S_{B 0}^{x}(0)\right\rangle=-\iota\left\langle e^{\iota H_{K} t / \hbar} c_{A 0} e^{-\iota\left(H_{K}+V_{x}\right) t / \hbar} c_{B 0}\right\rangle_{K},
\end{aligned}
$$

where the subscript $K$ for the expectation values indicates they are taken with respect to the ground state of $H_{K}$, and the bond potential reads $V_{x}=-2 \iota K c_{i} c_{j}$ with $\boldsymbol{i}$ and $\boldsymbol{j}$, again, representing the $A$ and its adjacent $x$-bond connected $B$ site, respectively [40,81].

The form of the correlators in Eq. (29) can be understood by taking into consideration the effect of operator $S_{i}^{\gamma}$ on an eigenstate. In addition to adding a single Majorana fermion $c_{i}$ at site $\boldsymbol{i}$, the spin operator also adds one $\pi$ flux each to the two plaquettes sharing the $\gamma$-bond emanating from $i$. The bond potential $V_{x}$ represents the insertion of this flux pair, and Eq. (29) calculates the dynamic rearrangement of the Majorana fermions at time $t$ following the sudden appearance of the fluxes at $t=0$. Incidentally, Baskaran et al. showed that this quench problem is equivalent to an exactly solvable $\mathrm{x}$-ray edge problem [81].

It was later shown by Knolle et al. [40] that due to a vanishing Majorana density of states in the Kitaev model, slowly switching on the bond potential in the infinite past and then switching it off in the infinite future-what may be called the adiabatic approximation-replicates the quench dynamics in the low-energy limit. Thus, as we desire a probe of the low-energy density of states, we perform the calculation under this approximation, meaning we rewrite Eqs. (29) so that the correlation functions are taken with respect to the Hamiltonian $H_{x}=H_{K}+V_{x}$, i.e., the Hamiltonian with a flux pair present, or equivalently one flipped $x$-bond. As a result, we evaluate

$$
\begin{aligned}
& \left\langle S_{A 0}^{x}(t) S_{A 0}^{x}(0)\right) \approx e^{\iota E_{0} t / \hbar}\left\langle c_{A 0} e^{-l H_{x} t / \hbar} c_{A 0}\right\rangle_{x}, \\
& \left\langle S_{A 0}^{x}(t) S_{B 0}^{x}(0)\right\rangle \approx-\iota e^{\ell E_{0} t / \hbar}\left\langle c_{A 0} e^{-\imath H_{x} t / \hbar} c_{B 0}\right\rangle_{x},
\end{aligned}
$$

where the subscript $x$ on the correlators explicitly indicates that they are now taken with respect to the ground state of $H_{x}$. Time-evolving the Majorana operators in Eqs. (30) in the 
Heisenberg picture $c_{i}(t)=e^{\imath H_{x} t / \hbar} c_{i} e^{-\imath H_{x} t / \hbar}$, we write

$$
\begin{aligned}
& \left\langle S_{A 0}^{x}(t) S_{A 0}^{x}(0)\right\rangle=e^{-\iota \Delta_{F} t / \hbar}\left\langle c_{A 0}(t) c_{A 0}(0)\right\rangle_{x}, \\
& \left\langle S_{A 0}^{x}(t) S_{B 0}^{x}(0)\right\rangle=-\iota e^{-\iota \Delta_{F} t / \hbar}\left\langle c_{A 0}(t) c_{B 0}(0)\right\rangle_{x},
\end{aligned}
$$

where the two-flux gap energy $\Delta_{F} \approx 0.26 K$ is the energy required to insert the fluxes [38].

Replacing the Majorana operators in the correlation functions with the complex fermions $b_{\boldsymbol{k}}$ allows us to rewrite the total susceptibility Eq. (28) as

$$
\chi(v)=-16 \iota N_{u} \int d t e^{\iota\left(\nu-\Delta_{F} / \hbar\right) t}\left\langle b_{0}(t) b_{0}^{\dagger}(0)\right\rangle_{x} .
$$

The last correlator can be obtained by solving a Dyson equation, details of which can be found in Appendix C. Utilizing the result, we produce (at zero temperature)

$$
\chi(v)=\frac{16 \hbar \theta\left(\hbar v-\Delta_{F}\right)\left[g_{11}^{R}\left(\hbar v-\Delta_{F}\right)-g_{11}^{A}\left(\hbar v-\Delta_{F}\right)\right]}{\left[1+\frac{4 K}{N_{u}} g_{11}^{R}\left(\hbar v-\Delta_{F}\right)\right]\left[1+\frac{4 K}{N_{u}} g_{11}^{A}\left(\hbar v-\Delta_{F}\right)\right]},
$$

where the local retarded Green function $g_{11}^{R}(\omega)$ is derived in Appendix $\mathrm{C}$ and is given by

$$
g_{11}^{R}(x)=\sum_{k} \frac{x+2 \varepsilon_{k}}{\left(x+\iota 0^{+}\right)^{2}-4\left|s_{k}\right|^{2}}=g_{11}^{A *}(x) .
$$

The ac noise correction generated in the metal by proximity to a gapless Kitaev spin liquid can now be calculated by utilizing Eq. (33) in Eq. (8) in the quantum limit. We find

$$
\begin{aligned}
S_{s}(\Omega, 0)= & \frac{32 \iota}{\hbar}\left(\frac{\mathcal{J} v_{0} m k_{F}}{2 \pi^{2} \hbar}\right)^{2} \int d \varepsilon(\hbar \Omega-\varepsilon) \theta(\hbar \Omega-\varepsilon) \\
& \times \frac{\theta\left(\varepsilon-\Delta_{F}\right)\left[g_{11}^{R}\left(\varepsilon-\Delta_{F}\right)-g_{11}^{A}\left(\varepsilon-\Delta_{F}\right)\right]}{\left[1+\frac{4 K}{N_{u}} g_{11}^{R}\left(\varepsilon-\Delta_{F}\right)\right]\left[1+\frac{4 K}{N_{u}} g_{11}^{A}\left(\varepsilon-\Delta_{F}\right)\right]},
\end{aligned}
$$

which can be measured electrically as ac resistance via Eq. (1).

Figure 6 depicts our numerical evaluation of Eq. (35) in the main plot, with the critical frequency $\Omega_{c}=\Delta_{F} / \hbar$ marked. The figure displays our primary result for the gapless Kitaev spin liquid compound, namely, that even in the ostensibly gapless case the ac noise correction generated in the metal is suppressed until frequencies greater than the two-flux gap, $\Delta_{F}$. Equivalently, via the FDT, measurement of the ac resistance enhancement in the metal near the critical frequency $\Omega_{c}$ should expose the two-flux gap when measured utilizing the proposed heterostructure.

That a completely suppressed region occurs seems odd given that the fermion spectrum $s_{k}$ is gapless, however while inserting a fermion into the lattice requires no extra energy input, it also requires the addition of a flux in each of the adjacent plquettes. These fluxes are gapped, and so an indirect gap appears even for the fermions. This outcome has been noted previously [40,77], however the proposed bilayer system offers a method for quantifying the flux gap experimentally.

\section{PHYSICAL ESTIMATES}

In this section, we estimate the expected signal strength of the voltage noise arising across the heavy metal layer of

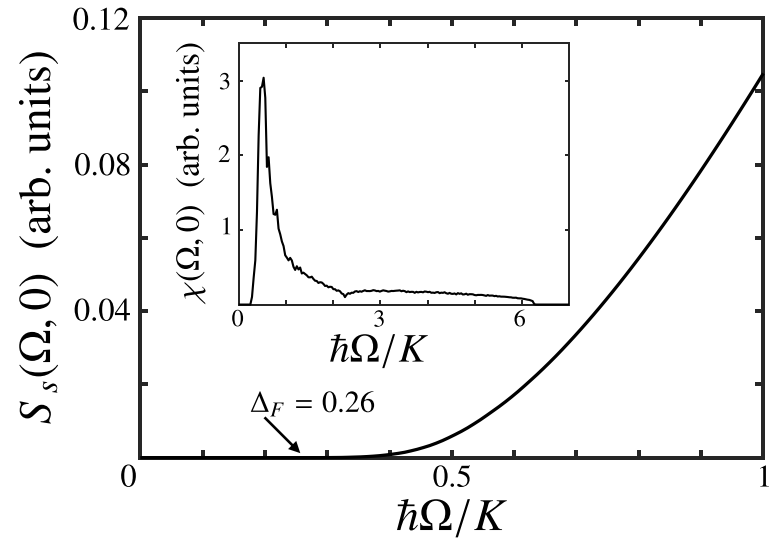

FIG. 6. A numerical plot showing $S_{s}(\Omega, 0)$ for the Kitaev model as a function of ac frequency normalized by the isotropic interaction strength, $K$. The main plot shows the computed sum. The graph is identically zero until a critical frequency equal to the gap energy is achieved, $\hbar \Omega_{c}=\Delta_{F} \approx 0.26 K$, at which point it becomes possible to inject flux pairs into the QSL and noise enhancement commences. Here, $N_{u}=4 \times 10^{4}$ has been used. The inset is the ac susceptibility $\chi(\Omega, 0)$ in the case of the Kitaev model, which can be extracted via numerical differentiation of Eq. (8), and is in good agreement with previous works [40].

our proposed bilayer system as a result of the proximity of a QSL state characterized by each of the three models we examine, noting that Eq. (1) connects the voltage noise and the ac resistance.

The variable $\Theta$ in Eq. (9) is a conversion parameter connecting the charge and spin sectors via the ISHE. We estimate the strength of $\Theta$ by first assuming, given $\mathrm{Pt}$ with a spin diffusion length $\lambda=2 \mathrm{~nm}$ and thickness $d=7 \mathrm{~nm}$, a spin current density profile of

$$
j_{s}(x, t)=N I_{s}(t) \frac{\sinh ((d-x) / \lambda)}{\sinh (d / \lambda)},
$$

where $N$ is the number of QSL spins per unit area on the interface and $I_{S}(t)$ is the spin current per QSL spin entering the interface. We model the spin to charge current conversion via the ISHE, so that we have $j_{c}(x, t)=\theta_{\mathrm{SH}}(2 e / \hbar) j_{s}(x, t)$, where $\theta_{\mathrm{SH}}$ is the spin Hall angle. Integrating over the cross-sectional area of the metal and multiplying by the resistivity $\rho$ reveals the total voltage generated across the metal:

$$
V_{c}(t)=\theta_{\mathrm{SH}}\left(\frac{2 e \lambda}{d \hbar}\right) N l \rho I_{s}(t) \tanh \left(\frac{d}{2 \lambda}\right),
$$

where $l$ is the length of the metal layer. Then we can reexpress Eq. (9) using voltage noise, $\delta S_{V}=\left\langle V_{c}(t) V_{c}(0)\right\rangle$, by writing

$$
\delta S_{V}(\Omega, T)=\left[\theta_{\mathrm{SH}} \frac{2 e}{\hbar} N \rho l \frac{\lambda}{d} \tanh \left(\frac{d}{2 \lambda}\right)\right]^{2} S_{S}(\Omega, T) .
$$

In $\operatorname{Pt} \theta_{\mathrm{SH}} \approx 0.1, \rho \approx 10^{-8} \Omega \mathrm{m}$, the lattice spacing $a=4 \AA \sim$ $k_{F}^{-1}$, and the electron effective mass $m=13 m_{e}$ [82]. The prefactor here characterizes $\Theta$.

In each QSL case, we assume an exchange coupling strength of $\mathcal{J} / k_{B} \sim 1 \mathrm{~K}$, set the unitless integral component of $S_{s} \sim 0.01$, and work out $N$ using the lattice spacing of the QSL spin sites. In the case of herbertsmithite, sample 
sizes can have linear dimensions of $1 \mathrm{~cm} \mathrm{[83],} \mathrm{and} \mathrm{the} \mathrm{lattice}$ spacing is on the order of $10 \AA$, so $N \sim 10^{20} \mathrm{spins} / \mathrm{m}^{2}$. Given that $J / k_{B} \sim 200 \mathrm{~K}[23]$ and using Eq. (12) in Eq. (38), our proposed bilayer system should find $\delta S_{V} \sim 10^{-18} V^{2} s$ when probing herbertsmithite.

For the organic triangular lattice compounds, sample sizes can have linear dimensions of $1 \mathrm{~mm}$ [18], and nearestneighbor spin distances on the order of $a \sim 10 \AA \sim k_{F s}^{-1}$ [18], so that $N \sim 10^{20}$ spins $/ \mathrm{m}^{2}$. We approximate the spinon velocity in the QSL using that of $1 \mathrm{D}$ spin chains, $v_{F s} \approx \pi J a / 2 \hbar$, as in Ref. [18], resulting in a Fermi frequency of $\Omega_{F s} \approx 10^{14} \mathrm{~Hz}$, and $J / k_{B} \sim 250 \mathrm{~K}[10]$ in these compounds. As a result, we find that $\delta S_{V}^{(0)} \sim 10^{-18} V^{2} s$, and the subleading correction due to the gauge field $\delta S_{V}^{(2)} \sim 10^{-20} V^{2} s$.

Lastly, we represent Kitaev compounds using $\alpha-\mathrm{RuCl}_{3}$, with a lattice constant of $a \approx 6 \AA$, sample sizes of linear dimension $1 \mathrm{~cm}$, and coupling strength $K / k_{B} \sim 80 \mathrm{~K}$ [26]. Thus $N \sim 10^{19}$ spins $/ \mathrm{m}^{2}$. Using Eq. (35) in Eq. (38) results in $\delta S_{V} \sim 10^{-18} V^{2} s$. Voltage fluctuations of these magnitudes have been measured in, e.g., Ref. [64].

\section{DISCUSSION AND CONCLUSIONS}

Equation (8) is a general relationship that quantifies the noise enhancement present in a normal metal adjacent to an insulating magnet lacking long-ranged magnetic order as a result of the coupling to that magnet, i.e., as a result of spin fluctuations across the interface. We assume that in the quantum limit the base ac resistance of a particular strongly spin-orbit coupled metal layer is known and constant, and as a result that background quantum noise can be accounted for and removed. This exposes the portion of the voltage noise power spectrum that quantifies the presence of the affixed quantum magnet, or more specifically the QSL candidate material. So, by measuring the ac frequency dependence of the spin fluctuations in the metal layer, it should be possible to compare the various QSL candidate materials in order to examine gapped and gapless models, and the low-energy spin excitations of these exotic phases of matter in general. Now we will connect the models we have examined to real materials.

The compound herbertsmithite, $\mathrm{ZnCu}_{3}(\mathrm{OH})_{6} \mathrm{Cl}_{2}$, evinces no long-range magnetic order down to temperatures of at least $50 \mathrm{mK}$, which is four orders of magnitude lower than its exchange coupling $J / k_{B} \sim 200 \mathrm{~K}$ [23], suggesting the presence of a QSL phase. Additionally, the $\mathrm{Cu}^{2+}$ ions form a perfect kagomé lattice, and therefore herbertsmithite is modeled, to a first approximation, by the HKLM. Experimental treatments of herbertsmithite include NMR [11,12], neutron scattering [24,25], and susceptibility [14] studies, most of which indicate that ground state excitations should be gapless. However, while some numerical DMRG studies agree [31], others show that the ground state of herbertsmithite should be a gapped spin liquid with $Z_{2}$ topological order [29], and the more recent NMR measurements indicate a gapped ground state as well [12]. Additional complications arise due to the fact that the low-energy spectrum appears to be dominated by impurity spins [84] that occur as a result of $\mathrm{Cu}^{2+}$ ions replacing some nonmagnetic $\mathrm{Zn}^{2+}$ in the transition metal sites between kagomé layers [85]. We have shown that our proposed heterostructure can address some of these issues. For instance, the presence of a gap energy will be indicated by a lack of frequency scaling in the voltage noise power spectrum measured in the heavy metal layer in the quantum limit up to a critical frequency of $\hbar \Omega_{c}=2 \Delta_{s}$. Technical issues notwithstanding, herbertsmithite remains one of the most promising QSL candidate material on offer, and our proposed technique adds a tool for further investigation.

The primary organic salt candidate QSL material that has been modeled as a spinon fermi sea coupled to an emergent $\mathrm{U}(1)$ gauge field is $\kappa$-(BEDT-TTF $)_{2}-\mathrm{Cu}_{2}(\mathrm{CN})_{3}$, henceforth $\kappa$-ET [32,33]. It is an antiferromagnetic weak Mott insulator, where structural dimers possessing a single spin-1/2 degree of freedom arise and form an approximately triangular lattice that becomes geometrically frustrated when cooled. The primary experimental evidence of QSL like behavior comes from thermal and NMR measurements; in the specific heat versus temperature, for example, a large linear term is observed [15-17], and NMR measurements show a lack of long-range magnetic ordering down to temperatures of $32 \mathrm{mK}$, approximately 4 orders of magnitude lower than the exchange coupling of $J / k_{B} \sim 250 \mathrm{~K}$ [10]. The necessity of working at very low temperatures when probing the low-energy density of states of $\kappa$-ET and similar compounds has caused some doubt as to the reliability of specific heat measurements, however, due to the difficulty of properly accounting for nuclear contributions. Nevertheless, other triangular lattice compounds exist, and the recent discoveries of possible QSL phases in $\mathrm{YbMgGaO}_{4}$ [86] and $\mathrm{NaYbO}_{2}$ [87], neither of which order down to temperatures of at least $70 \mathrm{mK}$, provide new opportunities to study previously unknown QSL candidate materials-opportunities the proposed heterostructure is intended to capitalize upon by providing a clear prediction for the frequency scaling of the modification to the ac resistance enhancement in the metal. We have shown that in its QSL state, a compound that can be modeled as a spinon fermi sea coupled to a U(1) gauge field should evince a correction to the ac resistance measured across a coupled heavy metal that goes as $\Omega^{4 / 3}$, which would therefore shed light on the low-energy density of states directly. The heterostructure proposed here would avoid the issue of contamination by signals due to nuclear spins entirely, and provide a powerful tool for probing the low-energy excitations of more recently discovered candidate QSL materials as well.

Finally, we mention the iridate honeycomb materials [88] and $\alpha-\mathrm{RuCl}_{3}$ [89] in the context of Kitaev compounds accessible to our bilayer technique. Initially, iridates like $\alpha-\mathrm{Na}_{2} \mathrm{IrO}_{3}$ and $\alpha-\mathrm{Li}_{2} \mathrm{IrO}_{3}$ were realized to have $\mathrm{Ir}^{4+}$ ions, with effective spin $j=1 / 2$, arranged in a honeycomb lattice. While these particular compounds were found to order at low temperatures [90-92], this class of materials does exhibit bond-directional Kitaev interactions [93]. Thus the discovery of a variant that does not magnetically order, $\mathrm{H}_{3} \mathrm{LiIr}_{2} \mathrm{O}_{6}$, is interesting [13]; in particular, it would be an excellent test of this material to see if the measured resistance enhancement in a coupled heavy metal film in fact behaves as we predict should be the case for Kitaev spin liquid compounds. A second material, $\alpha-\mathrm{RuCl}_{3}$, is also known to evince similar structural properties to the iridates, with magnetic $\mathrm{Ru}^{3+}$ ions forming a honeycomb lattice. 
As with most iridates, $\alpha-\mathrm{RuCl}_{3}$ orders at low temperatures [94], though interesting Kitaev spin liquidlike behavior occurs before ordering $[26,95]$. However, while $\alpha-\mathrm{RuCl}_{3}$ exhibits Kitaev-like physics prior to freezing, questions have arisen as to whether or not Kitaev physics are actually responsible for that appearance, rather than more conventional physics from the perspective of magnonlike excitations [96]. We have shown that the proposed bilayer could perhaps address this controversy directly, as the presence of Kitaev spin liquid physics in the $\alpha-\mathrm{RuCl}_{3}$ layer should result in characteristic suppression of the ac resistance enhancement in the coupled metal, particularly suppression below any flux gap present. We therefore believe that our proposed QSL-to-metal bilayer could be helpful to the search for a material exhibiting a true Kitaev spin liquid state.

In conclusion, we have proposed a heterostructure composed of a QSL candidate material overlaid with a strongly spin-orbit coupled heavy metal film as a viable probe of QSL ground states that can perhaps alleviate some of the currently outstanding controversies. Our proposal marries concepts from spintronics and quantum magnetism in order to use the well-understood physics of equilibrium noise in a new context, namely the search for, and categorization of, QSL ground states in various materials. The theory advanced here indicates that an equilibrium measurement, i.e., a measurement taken within the linear response regime, of the ac resistance in the normal metal layer will provide information about the lowenergy density of states of the QSL material under observation due to a connection via the FDT and Eq. (1) to the noise power spectrum we have calculated. In particular, determining the presence or absence of a gap, or probing a gapless QSL as in the $U(1)$ gauge fluctuations case will be readily accessible to this method. For quantifying a gap energy beyond the reach of modern electronic spectral analyzers, quantum absorption measurements utilizing the relaxation rate of a two level system coupled to the metal layer, as in Ref. [97], may prove advantageous. Thus the system we propose will have wide ranging applicability to probing insulating quantum magnets in general, and should provide an interesting method of examining and categorizing QSL candidate materials in particular. We show that the proposed bilayer should be able to extract any gap energy present in the HKLM, that fermions coupled to an emergent $\mathrm{U}(1)$ gauge field should see a subdominant frequency dependent correction arising in the ac resistance that goes as $\Omega^{4 / 3}$, and that the bare Kitaev model in the gapless spin liquid phase should nevertheless evince gapped behavior extricable through ac resistance measurements across the coupled metal layer.

We have restricted our analysis to bulk conversion effects in the metal layer, so in future works it could be interesting to consider interfacial effects that may affect conversion; in this vein examining different metal compounds like $\mathrm{CuBi}$ [98] with low intrinsic spin-orbit coupling could be illuminating. Additionally, it would be interesting to expand this analysis to include extensions to the QSL models such as Dzyaloshinskii-Moriya interactions, or correction to the Kitaev model [96], and especially to include disorder in the analysis, as disorder appears to be highly influential when attempting to discriminate between QSL and non-QSL ground states.

\section{ACKNOWLEDGMENTS}

We thank Gerrit E. W. Bauer and Yaroslav Tserkovnyak for discussions, and acknowledge support from The Research Foundation of The City University of New York, Fund \#90922-07 10.

\section{APPENDIX A: THE HEISENBERG KAGOMÉ LATTICE MODEL CALCULATION}

In this Appendix, we explicitly perform the steps involved in proceeding from Eq. (11) to Eq. (12) of the main text. The three basis vectors for the unit cell shown in Fig. 2 are

$$
\hat{\boldsymbol{e}}_{1}=\frac{a}{2}(1, \sqrt{3}), \quad \hat{\boldsymbol{e}}_{2}=\frac{a}{2}(1,-\sqrt{3}), \quad \hat{\boldsymbol{e}}_{3}=a(-1,0),
$$

where $a$ is the lattice constant. These unit vectors allow us to Fourier transform the Hamiltonian into momentum space, where we define st $k_{i}=\hat{\boldsymbol{e}}_{i} \cdot \boldsymbol{k}$. Equation (11) then reads

$$
H_{\mathrm{HKLM}}=\sum_{k} \Psi_{k}^{\dagger}\left(\begin{array}{cc}
\lambda & \mathrm{C}_{k}^{\dagger} \\
\mathrm{C}_{k} & \lambda
\end{array}\right) \Psi_{k},
$$

where $\Psi(\boldsymbol{k})=\left[b_{1}(\boldsymbol{k}), b_{2}(\boldsymbol{k}), b_{3}(\boldsymbol{k}), b_{1}^{\dagger}(-\boldsymbol{k}), b_{2}^{\dagger}(-\boldsymbol{k}), b_{3}^{\dagger}(-\boldsymbol{k})\right]^{T}$ is the vector of particle-hole boson operators for each of the three sites of the unit cell, and the matrix $\mathrm{C}_{\boldsymbol{k}}$ is a traceless $3 \times 3$ matrix with components

$$
\begin{aligned}
& c_{12}=J Q_{1} e^{i k_{1}}+J Q_{2} e^{-i k_{1}}=-c_{21}^{*}, \\
& c_{23}=J Q_{1} e^{i k_{2}}+J Q_{2} e^{-i k_{2}}=-c_{32}^{*}, \\
& c_{31}=J Q_{1} e^{i k_{3}}+J Q_{2} e^{-i k_{3}}=-c_{13}^{*} .
\end{aligned}
$$

Here, $Q_{1}, Q_{2} \in \mathbb{R}$ are the two distinct expectation values of $Q_{i j}$ (see Fig. 2), and as covered in the main text we explicitly consider the regime $Q_{1}=Q_{2}=Q$.

Diagonalizing Eq. (A2) with a unitary matrix $U$ leads to

$$
H_{\mathrm{HKLM}}=\sum_{k} \gamma_{k}^{\dagger} D \gamma_{k}
$$

with the rotated boson operators $\gamma_{k}=U^{\dagger} \Psi_{k}$. The diagonalized matrix $D=\operatorname{Diag}\left(\epsilon_{1}, \epsilon_{2}, \epsilon_{3}, \epsilon_{1}, \epsilon_{2}, \epsilon_{3}\right)$, where $\epsilon_{1}=\lambda$ and

$$
\epsilon_{2}=\epsilon_{3}=\sqrt{\lambda^{2}-4 J^{2} Q^{2}\left[\cos ^{2}\left(k_{1}\right)+\cos ^{2}\left(k_{2}\right)+\cos ^{2}\left(k_{3}\right)\right]} .
$$

To calculate the total spin susceptibility $\chi(v)=$ $\sum_{i}\left[\chi_{i i}^{+-}(v)+\chi_{i i}^{-+}(v)\right]$, we reexpress the sum over all lattice sites $i$ by reformulating it as $\sum_{i}=N_{u} \sum_{n=1}^{3}$, where $N_{u}$ is the number of unit cells and $n$ indexes each position within a single unit cell. We then obtain

$$
\begin{aligned}
\chi(v)= & -\iota \frac{2 \pi \hbar N_{u}}{N_{u}^{2}} \sum_{\boldsymbol{k}, \boldsymbol{q}} \sum_{n=1}^{3} \sum_{m, l=1}^{6} U_{n m}^{\boldsymbol{k} *} U_{n m}^{\boldsymbol{k}} U_{\bar{n} l}^{\boldsymbol{q} *} U_{\bar{n} l}^{\boldsymbol{q}} \\
& \times\left[\delta\left(\hbar v+\xi_{\boldsymbol{k} m}+\xi_{\boldsymbol{q} l}\right) \mathcal{N}_{m}(\boldsymbol{k}) \mathcal{N}_{l}(\boldsymbol{q})\right. \\
& \left.+\delta\left(\hbar v-\xi_{\boldsymbol{k} m}-\xi_{\boldsymbol{q} l}\right) \mathcal{M}_{m}(\boldsymbol{k}) \mathcal{M}_{l}(\boldsymbol{q})\right]
\end{aligned}
$$

where $U_{m l}^{k}$ is the $6 \times 6$ matrix that diagonalizes Eq. (11), $\bar{n}=n+3$, and $\xi_{k}=\left(\epsilon_{1}, \epsilon_{2}, \epsilon_{3},-\epsilon_{1},-\epsilon_{2},-\epsilon_{3}\right)$ is the vector 
of energy eigenvalues with the last three negated. Here, we also introduce vectors of distribution functions,

$$
\begin{gathered}
\mathcal{N}(\boldsymbol{k})=\left[n_{B}\left(\epsilon_{1}\right), n_{B}\left(\epsilon_{2}\right), n_{B}\left(\epsilon_{3}\right), \bar{n}_{B}\left(\epsilon_{1}\right), \bar{n}_{B}\left(\epsilon_{2}\right), \bar{n}_{B}\left(\epsilon_{3}\right)\right], \\
\mathcal{M}(\boldsymbol{k})=\left[\bar{n}_{B}\left(\epsilon_{1}\right), \bar{n}_{B}\left(\epsilon_{2}\right), \bar{n}_{B}\left(\epsilon_{3}\right), n_{B}\left(\epsilon_{1}\right), n_{B}\left(\epsilon_{2}\right), n_{B}\left(\epsilon_{3}\right)\right],
\end{gathered}
$$

where

$$
n_{B}(x)=\frac{1}{e^{\beta x}-1}
$$

and $\bar{n}_{B}=1+n_{B}$.
With this, we can express the ac, finite temperature noise created by the proximate QSL by using Eq. (A4) in Eq. (8),

$$
\begin{aligned}
S_{S}(\Omega, T)= & \frac{4 \pi}{\hbar N_{u}}\left(\frac{\mathcal{J} v_{0} m k_{F}}{2 \pi^{2} \hbar}\right)^{2} \sum_{\boldsymbol{k}, \boldsymbol{q}} \sum_{n, m, l} U_{n m}^{\boldsymbol{k} *} U_{n m}^{\boldsymbol{k}} U_{\bar{n} l}^{\boldsymbol{q} *} U_{\bar{n} l}^{\boldsymbol{q}} \\
& \times\left[\frac{-\xi_{\boldsymbol{k} m}-\xi_{\boldsymbol{q} l}-\hbar \Omega}{e^{-\beta\left(\xi_{\boldsymbol{k} m}+\xi_{\boldsymbol{q} l}+\hbar \Omega\right)}-1} \mathcal{N}_{m}(\boldsymbol{k}) \mathcal{N}_{l}(\boldsymbol{q})\right. \\
& \left.+\frac{\xi_{k m}+\xi_{\boldsymbol{q} l}-\hbar \Omega}{e^{\beta\left(\xi_{k m}+\xi_{\boldsymbol{q} l}-\hbar \Omega\right)}-1} \mathcal{M}_{m}(\boldsymbol{k}) \mathcal{M}_{l}(\boldsymbol{q})\right]
\end{aligned}
$$

The zero temperature limit of this result gives Eq. (12).

\section{APPENDIX B: U(1) GAUGE FIELD CORRECTION TO THE SUSCEPTIBILITY}

In this Appendix, we examine the steps involved in proceeding from Eq. (19) to Eq. (20). When considering a single gauge propagator, there are three contributing diagrams as shown in Fig. 4. Figures 4(a) and 4(b), where the gauge propagator does not cross the particle-hole bubble, are self-energy corrections to a fermion line, and Fig. 4(c), where the propagator does cross the bubble, is a vertex correction. Maintaining the gauge invariance of the susceptibility requires that all three diagrams are included in the calculation $[75,76]$.

We begin this section with the action given in Eq. (13) of the main text, and place it explicitly on the Keldysh contour in the Keldysh basis. We write the components of the action zeroth and first order in the gauge field as

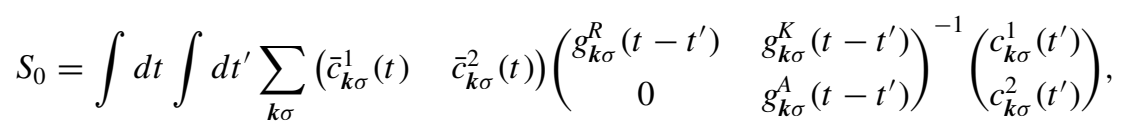

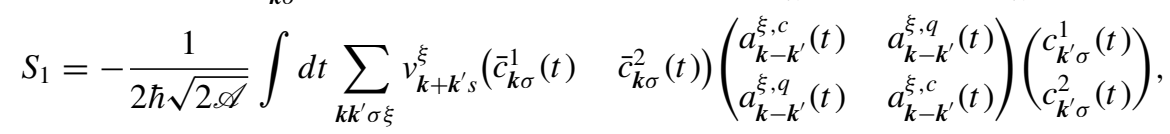

where $v_{\boldsymbol{k} s}=\hbar \boldsymbol{k} / m_{s}$. We have split the gauge field into its classical and quantum components denoted by the $c$ and $q$ superscripts, and split the fermion fields into their 1 and 2 components in accordance with Ref. [99]. Here, $S_{1}$ is the RAK basis equivalent to what we have called $S_{\text {int }}$ in the main text.

Expanding Eq. (19), and restricting ourselves to terms with a single gauge propagator, it is possible to write three expressions of the form $\chi_{n}^{(2)}(\boldsymbol{p}, v)=\chi_{n}^{+-(2)}(\boldsymbol{p}, v)+\chi_{n}^{-+(2)}(\boldsymbol{p}, v)$, each corresponding to one of the diagrams in Fig. 4:

$$
\begin{aligned}
\chi_{a}^{(2)}(\boldsymbol{p}, v)= & \frac{1}{4 \hbar^{2} \mathscr{A}^{2}} \sum_{\boldsymbol{k} \boldsymbol{q}} \sum_{\xi \xi^{\prime}} \sum_{\eta_{1} \eta_{2}} \int \frac{d \Omega}{2 \pi} \int \frac{d \omega}{2 \pi} \eta_{1} \eta_{2} D_{\xi \xi^{\prime}}^{\eta_{1} \eta_{2}}(-\boldsymbol{q},-\omega) v_{2 \boldsymbol{k}+2 \boldsymbol{p}+\boldsymbol{q} s}^{\xi^{\prime}} v_{2 \boldsymbol{k}+2 \boldsymbol{p}+\boldsymbol{q} s}^{\xi^{\prime}} \\
& \times\left[g_{\boldsymbol{k}+\boldsymbol{p}}^{-\eta_{1}}(\Omega+v) g_{\boldsymbol{k}+\boldsymbol{p}+\boldsymbol{q}}^{\eta_{1} \eta_{2}}(\Omega+v+\omega) g_{\boldsymbol{k}+\boldsymbol{p}}^{\eta_{2}+}(\Omega+v) g_{\boldsymbol{k}}^{+-}(\Omega)\right], \\
\chi_{b}^{(2)}(\boldsymbol{p}, v)= & \frac{1}{4 \hbar^{2} \mathscr{A}^{2}} \sum_{\boldsymbol{k} \boldsymbol{q}} \sum_{\xi \xi^{\prime}} \sum_{\eta_{1} \eta_{2}} \int \frac{d \Omega}{2 \pi} \int \frac{d \omega}{2 \pi} \eta_{1} \eta_{2} D_{\xi \xi^{\prime}}^{\eta_{1} \eta_{2}}(-\boldsymbol{q},-\omega) v_{2 \boldsymbol{k}+2 \boldsymbol{p}+\boldsymbol{q} s^{\xi}}^{\xi_{2}} v_{2+2 \boldsymbol{p}+\boldsymbol{q} s}^{\xi^{\prime}} \\
& \times\left[g_{\boldsymbol{k}}^{-+}(\Omega+v) g_{\boldsymbol{k}+\boldsymbol{p}}^{+\eta_{1}}(\Omega) g_{\boldsymbol{k}+\boldsymbol{p}+\boldsymbol{q}}^{\eta_{1} \eta_{2}}(\Omega+\omega) g_{\boldsymbol{k}+\boldsymbol{p}}^{\eta_{2}-}(\Omega)\right], \\
\chi_{c}^{(2)}(\boldsymbol{p}, v)= & \frac{1}{4 \hbar^{2} \mathscr{A}^{2}} \sum_{\boldsymbol{k} \boldsymbol{q}} \sum_{\xi \xi^{\prime}} \sum_{\eta_{1} \eta_{2}} \int \frac{d \Omega}{2 \pi} \int \frac{d \omega}{2 \pi} \eta_{1} \eta_{2} D_{\xi \xi^{\prime}}^{\eta_{1} \eta_{2}}(-\boldsymbol{q},-\omega) v_{2 \boldsymbol{k}+\boldsymbol{q} s}^{\xi^{\prime}} v_{2 \boldsymbol{k}+2 \boldsymbol{p}+\boldsymbol{q} s}^{\xi^{\prime}} \\
& \times\left[g_{\boldsymbol{k}+\boldsymbol{p}}^{-\eta_{1}}(\Omega+v) g_{\boldsymbol{k}+\boldsymbol{p}+\boldsymbol{q}}^{\eta_{1}+}(\Omega+v+\omega) g_{\boldsymbol{k}+\boldsymbol{q}}^{+\eta_{2}}(\Omega+\omega) g_{\boldsymbol{k}}^{\eta_{2}-}(\Omega)\right] .
\end{aligned}
$$

Here, $\eta_{1}, \eta_{2}= \pm$ represent Keldysh indices for the forward and backward contours, and bare \pm superscripts on the Green functions also represent the externally fixed forward and backward Keldysh indices. The gauge propagator, which can be derived from the Matsubara formalism in the Coulomb gauge by analytic continuation of the zero temperature result, is given as

$$
D_{\xi \xi^{\prime}}^{R, A}(\boldsymbol{q}, \omega)=-\left(\delta_{\xi \xi^{\prime}}-\frac{q_{\xi} q_{\xi^{\prime}}}{q^{2}}\right) d_{\boldsymbol{q}}^{R, A}(\omega), \text { with } d_{\boldsymbol{q}}^{R, A}(\omega)=\frac{1}{N} \frac{1}{\chi_{d} q^{2} \mp \iota \frac{\omega E_{F}}{v_{F} q \pi \hbar^{3}}},
$$

where $E_{F}$ is the Fermi energy, and $\chi_{d}=\left(24 \pi \hbar m_{s}\right)^{-1}$ is the Landau diamagnetic susceptibility of the fermions.

We now introduce the fermion self-energy in order to represent Eqs. (B3) and (B4) in terms of the self-energy. Note at the outset that because the system we consider is in equilibrium, only the retarded spinon self-energy need be explicitly constructed; the advanced self-energy can be derived from the retarded term. The retarded one-loop self-energy can be calculated 
by considering the second-order correction to the retarded Green function using $S_{1}$ as a perturbation

$$
g_{\boldsymbol{k} \sigma}^{R(2)}\left(t, t^{\prime}\right)=\frac{\iota}{2}\left\langle c_{\boldsymbol{k} \sigma}^{1}(t) \bar{c}_{\boldsymbol{k} \sigma}^{1}\left(t^{\prime}\right) S_{1}^{2}\right\rangle .
$$

Extracting the self-energy is then a matter of expanding this quantity and enforcing causality, resulting in

$$
\Sigma_{\boldsymbol{k} \sigma}^{R}\left(t, t^{\prime}\right)=\frac{2 \iota}{(4 \hbar)^{2} \mathscr{A}} \sum_{\boldsymbol{q}} \sum_{\xi \xi^{\prime}}\left[g_{\boldsymbol{k}+\boldsymbol{q} \sigma}^{R}\left(t-t^{\prime}\right) D_{\xi \xi^{\prime}}^{K}\left(-\boldsymbol{q}, t-t^{\prime}\right)+g_{\boldsymbol{k}+\boldsymbol{q} \sigma}^{K}\left(t-t^{\prime}\right) D_{\xi \xi^{\prime}}^{R}\left(-\boldsymbol{q}, t-t^{\prime}\right)\right] v_{2 \boldsymbol{k}+\boldsymbol{q} s}^{\xi} v_{2 \boldsymbol{k}+\boldsymbol{q} s^{\prime}}^{\xi^{\prime}}
$$

Completing the $\xi$ and $\xi^{\prime}$ sums and transforming to frequency space, we have

$$
\Sigma_{\boldsymbol{k} \sigma}^{R}(\Omega)=-\frac{\iota}{2 \mathscr{A}} \int \frac{d \omega}{2 \pi} \sum_{\boldsymbol{q}}\left(\frac{|\boldsymbol{k} \times \hat{\boldsymbol{q}}|}{m_{s}}\right)^{2}\left[g_{\boldsymbol{k}+\boldsymbol{q} \sigma}^{R}(\Omega+\omega) d_{-\boldsymbol{q}}^{K}(-\omega)+g_{\boldsymbol{k}+\boldsymbol{q} \sigma}^{K}(\Omega+\omega) d_{-\boldsymbol{q}}^{R}(-\omega)\right] .
$$

In general, we expect $d_{-q}(-\omega)$ to be dominated by small $\boldsymbol{q}$, due to the form of the propagator. By selecting the coordinates $\boldsymbol{q}=q_{\|} \hat{\boldsymbol{k}}+\hat{z} \times \hat{\boldsymbol{k}} q_{\perp}$ we can express the dispersion as

$$
\zeta_{k+q} \approx \epsilon_{k}-\mu+\frac{\hbar^{2} q_{\perp}^{2}}{2 m_{s}}+\hbar v_{F s} q_{\|},
$$

with $\epsilon_{\boldsymbol{k}}=\hbar^{2} k^{2} / 2 m_{s}$. From this, we further expect to find momentum scaling as $q_{\|} \sim q_{\perp}^{2} \ll q_{\perp}$, i.e., where $\boldsymbol{q}$ is essentially normal to $\boldsymbol{k}$, which allows us to replace the momentum dependence of the gauge propagator with $q_{\perp}$ and write $|\boldsymbol{k} \times \hat{\boldsymbol{q}}|^{2} \approx k_{F S}^{2}$ for momentum near the spinon Fermi surface. Therefore, by explicitly substituting in the spinon Green functions, Eq. (B9) becomes

$$
\begin{aligned}
\Sigma_{k \sigma}^{R}(\Omega) \approx & -\frac{\iota k_{F s}^{2} a_{s}}{2 m_{s}^{2}} \int \frac{d \omega}{2 \pi} \int \frac{d q_{\perp}}{2 \pi} \int \frac{d q_{\|}}{2 \pi}\left[\frac{d_{-q_{\perp}}^{K}(-\omega)}{\Omega+\omega-\frac{\zeta_{k}}{\hbar}-\frac{\epsilon_{q_{\perp}}}{\hbar}-v_{F s} q_{\|}+\iota \delta}-2 \pi \iota \delta\left(\Omega+\omega-\frac{\zeta_{k}}{\hbar}-\frac{\epsilon_{q_{\perp}}}{\hbar}-v_{F s} q_{\|}\right)\right. \\
& \left.\times \tanh \left(\frac{\hbar(\Omega+\omega)}{2 k_{B} T}\right) d_{q_{\perp}}^{R}(-\omega)\right] .
\end{aligned}
$$

At this point, it is clear that the $q_{\|}$integral poses no trouble, so we complete it and find

$$
\Sigma_{k \sigma}^{R}(\Omega) \approx \frac{v_{F s} a_{s}}{2 \hbar^{2}} \int \frac{d \omega}{2 \pi} \int \frac{d q_{\perp}}{2 \pi}\left[-\frac{1}{2} \operatorname{coth}\left(\frac{\hbar \omega}{2 k_{B} T}\right)\left[d_{q_{\perp}}^{R}(-\omega)-d_{q_{\perp}}^{A}(-\omega)\right]+\tanh \left(\frac{\hbar(\Omega+\omega)}{2 k_{B} T}\right) d_{q_{\perp}}^{R}(-\omega)\right] .
$$

Note that from this expression for the self-energy, we can see that the $\boldsymbol{k}$ dependence drops out entirely.

Evaluating the sums over the Keldysh indices in the self-energy correction terms, Eqs. (B3) and (B4), allows us to rewrite the expressions for diagrams $a$ and $b$ in terms of spinon Green's functions and the self-energy:

$$
\begin{aligned}
\chi_{a}^{(2)}(\boldsymbol{p}, v)= & -\iota \frac{1}{1-e^{-\beta \hbar v}} \frac{1}{2 \mathscr{A}} \sum_{\boldsymbol{k}} \int \frac{d \Omega}{2 \pi}\left[\tanh \left(\frac{\hbar \Omega}{2 k_{B} T}\right)-\tanh \left(\frac{\hbar(\Omega+v)}{2 k_{B} T}\right)\right] \\
& \times\left[g_{k}^{R}(\Omega)-g_{\boldsymbol{k}}^{A}(\Omega)\right]\left[g_{\boldsymbol{k}+\boldsymbol{p}}^{R}(\Omega+v) \Sigma_{\boldsymbol{k}+\boldsymbol{p}}^{R}(\Omega+v) g_{\boldsymbol{k}+\boldsymbol{p}}^{R}(\Omega+v)-g_{\boldsymbol{k}+\boldsymbol{p}}^{A}(\Omega+v) \Sigma_{\boldsymbol{k}+\boldsymbol{p}}^{A}(\Omega+v) g_{\boldsymbol{k}+\boldsymbol{p}}^{A}(\Omega+v)\right], \\
\chi_{b}^{(2)}(\boldsymbol{p}, v)= & -\iota \frac{1}{1-e^{-\beta \hbar v}} \frac{1}{2 \mathscr{A}} \sum_{\boldsymbol{k}} \int \frac{d \Omega}{2 \pi}\left[\tanh \left(\frac{\hbar \Omega}{2 k_{B} T}\right)-\tanh \left(\frac{\hbar(\Omega+v)}{2 k_{B} T}\right)\right] \\
& \times\left[g_{k+p}^{R}(\Omega+v)-g_{\boldsymbol{k}+\boldsymbol{p}}^{A}(\Omega+v)\right]\left[g_{\boldsymbol{k}}^{R}(\Omega) \Sigma_{\boldsymbol{k}}^{R}(\Omega) g_{\boldsymbol{k}}^{R}(\Omega)-g_{\boldsymbol{k}}^{A}(\Omega) \Sigma_{\boldsymbol{k}}^{A}(\Omega) g_{\boldsymbol{k}}^{A}(\Omega)\right] .
\end{aligned}
$$

In the end, we must sum together all three diagrams in order to maintain gauge invariance, which we begin by combining these two terms into $\chi_{a b}^{(2)}(\boldsymbol{p}, v)=\chi_{a}^{(2)}(\boldsymbol{p}, v)+\chi_{b}^{(2)}(\boldsymbol{p}, v)$, and use the fact that any integral over only retarded or advanced terms vanishes, to write $\chi_{a b}^{(2)}(\boldsymbol{p}, v)$ compactly. The outcome is

$$
\begin{aligned}
\chi_{a b}^{(2)}(\boldsymbol{p}, v)= & \iota \frac{m_{s} a_{s}}{2 \hbar} \frac{1}{1-e^{-\beta \hbar \nu}} \int \frac{d \Omega}{2 \pi} \int_{-\mu}^{\infty} \frac{d \zeta_{\boldsymbol{k}}}{2 \pi} \int \frac{d \theta_{\boldsymbol{k}}}{2 \pi}\left[\tanh \left(\frac{\hbar \Omega}{2 k_{B} T}\right)-\tanh \left(\frac{\hbar(\Omega+v)}{2 k_{B} T}\right)\right] \\
& \times\left[\frac{g_{\boldsymbol{k}}^{A}(\Omega)\left[\Sigma_{\boldsymbol{k}+\boldsymbol{p}}^{R}(\Omega+v)-\Sigma_{\boldsymbol{k}}^{A}(\Omega)\right] g_{\boldsymbol{k}+\boldsymbol{p}}^{R}(\Omega+v)}{\nu+\frac{\zeta_{k}}{\hbar}-\frac{\zeta_{\boldsymbol{k}+p}}{\hbar}+\iota \delta}+\frac{g_{\boldsymbol{k}}^{R}(\Omega)\left[\Sigma_{\boldsymbol{k}+\boldsymbol{p}}^{A}(\Omega+v)-\Sigma_{\boldsymbol{k}}^{R}(\Omega)\right] g_{\boldsymbol{k}+\boldsymbol{p}}^{A}(\Omega+v)}{v+\frac{\zeta_{k}}{\hbar}-\frac{\zeta_{\boldsymbol{k}+p}}{\hbar}-\iota \delta}\right],
\end{aligned}
$$

where we have represented the $\boldsymbol{k}$ integral in polar coordinates, with $\theta_{\boldsymbol{k}}$ the angle between $\boldsymbol{k}$ and $\boldsymbol{p}$, and performed a change of variables from $k$ to $\zeta_{k}$. Here it is important to point out two things: first, the Fermi surface $\mu$ is the largest energy scale in the problem, and so we extend the lower bound of the $\zeta_{k}$ integral to $-\infty$. Second, note that $\zeta_{k+p}-\zeta_{k}=\epsilon_{p}+\hbar v_{F s} p \cos \theta_{k} \approx$ $\hbar v_{F s} p \cos \theta_{\boldsymbol{k}}$, where the last step is due to the expectation that small $p$ dominates, which means the $\zeta_{\boldsymbol{k}}$ dependence drops out of the denominator in both terms. Thus there is no $\zeta_{k}$ dependence in either the denominator or the self-energies, and so we can 
complete the $\zeta_{\boldsymbol{k}}$ integral by simply integrating over the spinon Green functions; this is the reason terms that only have retarded or advanced Green functions in them vanish. The result is

$$
\begin{aligned}
\chi_{a b}^{(2)}(\boldsymbol{p}, v)= & -\frac{m_{s} a_{s}}{2 \hbar} \frac{1}{1-e^{-\beta \hbar v}} \int \frac{d \Omega}{2 \pi} \int \frac{d \theta_{\boldsymbol{k}}}{2 \pi}\left[\tanh \left(\frac{\hbar \Omega}{2 k_{B} T}\right)-\tanh \left(\frac{\hbar(\Omega+v)}{2 k_{B} T}\right)\right] \\
& \times\left[\frac{\Sigma_{\boldsymbol{k}+\boldsymbol{p}}^{R}(\Omega+v)-\Sigma_{\boldsymbol{k}}^{A}(\Omega)}{\left(v-v_{F s} p \cos \theta_{\boldsymbol{k}}+\iota \delta\right)^{2}}-\frac{\Sigma_{\boldsymbol{k}+\boldsymbol{p}}^{A}(\Omega+v)-\Sigma_{\boldsymbol{k}}^{R}(\Omega)}{\left(v-v_{F s} p \cos \theta_{\boldsymbol{k}}-\iota \delta\right)^{2}}\right] .
\end{aligned}
$$

We can now substitute in the self-energies represented in Eq. (B12) to present the final step in combining these two diagrams,

$$
\begin{aligned}
\chi_{a b}^{(2)}(\boldsymbol{p}, v)= & -\frac{k_{F s} a_{s}^{2}}{2 \hbar^{2}} \frac{1}{1-e^{-\beta \hbar v}} \int \frac{d \Omega}{2 \pi} \int \frac{d \omega}{2 \pi} \int \frac{d \theta_{\boldsymbol{k}}}{2 \pi} \int \frac{d q_{\perp}}{2 \pi}\left[\tanh \left(\frac{\hbar(\Omega+v)}{2 k_{B} T}\right)-\tanh \left(\frac{\hbar \Omega}{2 k_{B} T}\right)\right] \\
& \times\left\{\frac{\operatorname{coth}\left(\frac{\hbar \omega}{2 k_{B} T}\right)\left[d_{q_{\perp}}^{R}(-\omega)-d_{q_{\perp}}^{A}(-\omega)\right]-\tanh \left(\frac{\hbar(\Omega+v+\omega)}{2 k_{B} T}\right) d_{q_{\perp}}^{R}(-\omega)+\tanh \left(\frac{\hbar(\Omega+\omega)}{2 k_{B} T}\right) d_{q_{\perp}}^{A}(-\omega)}{\left(v-v_{F s} p \cos \theta_{\boldsymbol{k}}+\iota \delta\right)^{2}}\right. \\
& \left.+\frac{\operatorname{coth}\left(\frac{\hbar \omega}{2 k_{B} T}\right)\left[d_{q_{\perp}}^{R}(-\omega)-d_{q_{\perp}}^{A}(-\omega)\right]+\tanh \left(\frac{\hbar(\Omega+v+\omega}{2 k_{B} T}\right) d_{q_{\perp}}^{A}(-\omega)-\tanh \left(\frac{\hbar(\Omega+\omega)}{2 k_{B} T}\right) d_{q_{\perp}}^{R}(-\omega)}{\left(v-v_{F s} p \cos \theta_{k}-\iota \delta\right)^{2}}\right\} .
\end{aligned}
$$

The third diagram corresponds to a vertex correction. We expect that $p \ll k$ dominates, and recall that the form of the gauge propagator restricts us to small $q$ as well. We express $\boldsymbol{q}$ in components perpendicular and parallel to $\boldsymbol{k}$ as above, and note that, via the same approximation as in Ref. [76], near the Fermi surface we can write

$$
-\sum_{\xi \xi^{\prime}}\left(\delta_{\xi \xi^{\prime}}-\frac{q_{\xi} q_{\xi^{\prime}}}{q^{2}}\right) v_{2 \boldsymbol{k}+\boldsymbol{q} s}^{\xi} v_{2 \boldsymbol{k}+2 \boldsymbol{p}+\boldsymbol{q} s}^{\xi^{\prime}} \approx-\frac{4 \hbar^{2} k_{F s}^{2}}{m_{s}^{2}}\left(\frac{q_{\perp}^{2}}{q^{2}}+\frac{q_{\|}^{2}}{q^{2}}-\frac{q_{\|} q_{\perp}}{q^{2}}\right) \approx-4 v_{F s}^{2} .
$$

Above, the first approximation is from recalling that $p \ll k$ and the second is a result of $q_{\|} \sim q_{\perp}^{2} \ll q_{\perp}$. Therefore summing over $\xi$ and $\xi^{\prime}$ and expanding the Keldysh indices, Eq. (B5) becomes

$$
\begin{aligned}
\chi_{c}^{(2)}(\boldsymbol{p}, v) & =-\frac{v_{F}^{2}}{2 \hbar^{2} \mathscr{A}^{2}} \frac{1}{1-e^{-\beta \hbar v}} \sum_{\boldsymbol{k} \boldsymbol{q}} \int \frac{d \Omega}{2 \pi} \int \frac{d \omega}{2 \pi}\left[\tanh \left(\frac{\hbar(\Omega+v)}{2 k_{B} T}\right)-\tanh \left(\frac{\hbar \Omega}{2 k_{B} T}\right)\right] \\
& \times\left\{g _ { \boldsymbol { k } + \boldsymbol { p } } ^ { R } ( \Omega + v ) g _ { \boldsymbol { k } + \boldsymbol { p } + \boldsymbol { q } } ^ { R } ( \Omega + v + \omega ) g _ { \boldsymbol { k } + \boldsymbol { q } } ^ { A } ( \Omega + \omega ) g _ { \boldsymbol { k } } ^ { A } ( \Omega ) \left[\operatorname{coth}\left(\frac{\hbar v}{2 k_{B} T}\right)\left[d_{q_{\perp}}(-\omega)-d_{q_{\perp}}(-\omega)\right]\right.\right. \\
& \left.-d_{q_{\perp}}^{R}(-\omega) \tanh \left(\frac{\hbar(\Omega+v+\omega)}{2 k_{B} T}\right)+d_{q_{\perp}}^{A}(-\omega) \tanh \left(\frac{\hbar(\Omega+\omega)}{2 k_{B} T}\right)\right] \\
& +g_{\boldsymbol{k}+\boldsymbol{p}}^{A}(\Omega+v) g_{\boldsymbol{k}+\boldsymbol{p}+\boldsymbol{q}}^{A}(\Omega+v+\omega) g_{\boldsymbol{k}+\boldsymbol{q}}^{R}(\Omega+\omega) g_{\boldsymbol{k}}^{R}(\Omega)\left[\operatorname{coth}\left(\frac{\hbar v}{2 k_{B} T}\right)\left[d_{q_{\perp}}(-\omega)-d_{q_{\perp}}(-\omega)\right]\right. \\
& \left.\left.+d_{q_{\perp}}^{A}(-\omega) \tanh \left(\frac{\hbar(\Omega+v+\omega)}{2 k_{B} T}\right)-d_{q_{\perp}}^{R}(-\omega) \tanh \left(\frac{\hbar(\Omega+\omega)}{2 k_{B} T}\right)\right]\right\}
\end{aligned}
$$

Representing the $\boldsymbol{k}$ integral in polar coordinates, where again the angle is with respect to the momentum $\boldsymbol{p}$, and then performing a change of variables from the modulus $k$ to $\zeta_{k}$, we can complete the $q_{\|}$and $\zeta_{k}$ integrals. Note that the only quantities that contain these variables now are the spinon Green's functions, and those only occur only in specific pairings. We find

$$
\begin{gathered}
\int \frac{d \zeta_{\boldsymbol{k}}}{2 \pi} \int \frac{d q_{\|}}{2 \pi} g_{\boldsymbol{k}+\boldsymbol{p}}^{R}(\Omega+v) g_{\boldsymbol{k}+\boldsymbol{p}+\boldsymbol{q}}^{R}(\Omega+v+\omega) g_{\boldsymbol{k}+\boldsymbol{q}}^{A}(\Omega+\omega) g_{\boldsymbol{k}}^{A}(\Omega) \\
=\frac{-k_{F s} / v_{F s}^{2}}{\left(v-v_{F s} p \cos \theta_{\boldsymbol{k}}+\iota \delta\right)\left(v-v_{F s} p \cos \theta_{\boldsymbol{k}}-\frac{\hbar}{m_{s}} q_{\perp} p \sin \theta_{k}+\iota \delta\right)} \\
\int \frac{d \zeta_{\boldsymbol{k}}}{2 \pi} \int \frac{d q_{\|}}{2 \pi} g_{\boldsymbol{k}+\boldsymbol{p}}^{A}(\Omega+v) g_{\boldsymbol{k}+\boldsymbol{p}+\boldsymbol{q}}^{A}(\Omega+v+\omega) g_{\boldsymbol{k}+\boldsymbol{q}}^{R}(\Omega+\omega) g_{\boldsymbol{k}}^{R}(\Omega) \\
=\frac{-k_{F s} / v_{F s}^{2}}{\left(v-v_{F s} p \cos \theta_{\boldsymbol{k}}-\iota \delta\right)\left(v-v_{F s} p \cos \theta_{\boldsymbol{k}}-\frac{\hbar}{m_{s}} q_{\perp} p \sin \theta_{k}-\iota \delta\right)}
\end{gathered}
$$


which we can use in $\chi_{c}^{(2)}(\boldsymbol{p}, v)$. The result is

$$
\begin{aligned}
\chi_{c}^{(2)}(\boldsymbol{p}, v) & =\frac{k_{F s} a_{s}^{2}}{2 \hbar^{2}} \frac{1}{1-e^{-\beta \hbar \nu}} \int \frac{d \Omega}{2 \pi} \int \frac{d \omega}{2 \pi} \int \frac{d \theta_{\boldsymbol{k}}}{2 \pi} \int \frac{d q_{\perp}}{2 \pi}\left[\tanh \left(\frac{\hbar(\Omega+v)}{2 k_{B} T}\right)-\tanh \left(\frac{\hbar \Omega}{2 k_{B} T}\right)\right] \\
& \times\left[\frac{\operatorname{coth}\left(\frac{\hbar v}{2 k_{B} T}\right)\left[d_{q_{\perp}}^{R}(-\omega)-d_{q_{\perp}}^{A}(-\omega)\right]-d_{q_{\perp}}^{R}(-\omega) \tanh \left(\frac{\hbar(\Omega+v+\omega)}{2 k_{B} T}\right)+d_{q_{\perp}}^{A}(-\omega) \tanh \left(\frac{\hbar(\Omega+\omega)}{2 k_{B} T}\right)}{\left(v-v_{F s} p \cos \theta_{k}+\iota \delta\right)\left(v-v_{F s} p \cos \theta_{k}-\frac{\hbar}{m_{s}} q_{\perp} p \sin \theta_{k}+\iota \delta\right)}\right. \\
& \left.+\frac{\operatorname{coth}\left(\frac{\hbar v}{2 k_{B} T}\right)\left[d_{q_{\perp}}^{R}(-\omega)-d_{q_{\perp}}^{A}(-\omega)\right]+d_{q_{\perp}}^{A}(-\omega) \tanh \left(\frac{\hbar(\Omega+v+\omega)}{2 k_{B} T}\right)-d_{q_{\perp}}^{R}(-\omega) \tanh \left(\frac{\hbar(\Omega+\omega)}{2 k_{B} T}\right)}{\left(v-v_{F s} p \cos \theta_{k}-\iota \delta\right)\left(v-v_{F s} p \cos \theta_{k}-\frac{\hbar}{m_{s}} q_{\perp} p \sin \theta_{k}-\iota \delta\right)}\right],
\end{aligned}
$$

which we must combine with Eq. (B17) in order to maintain gauge invariance.

In order to express the combined term more concisely, we perform the angular integral and introduce the function

$$
\begin{aligned}
I^{ \pm}(q) & =\int \frac{d \theta_{\boldsymbol{k}}}{2 \pi} \frac{1}{x-\cos \theta_{\boldsymbol{k}} \pm \iota \delta} \frac{1}{x-\cos \theta_{\boldsymbol{k}}-\frac{q}{k_{F}} \sin \theta_{k} \pm \iota \delta} \\
& =\frac{|x|}{(x \pm \iota \delta)^{2} \sqrt{(x \pm \iota \delta)^{2}-\left(\left(\frac{q}{k_{F}}\right)^{2}+1\right)}}
\end{aligned}
$$

where $x=v /\left(v_{F s} p\right)$ and we have substituted $q_{\perp} \rightarrow q$ for notational convenience. Therefore the sum of Eq. (B17) and Eq. (B20) becomes

$$
\begin{aligned}
\chi^{(2)}(\boldsymbol{p}, v)= & \frac{k_{F s} a_{s}^{2}}{2 \hbar^{2}} \frac{1}{\left(v_{F s} p\right)^{2}} \frac{1}{1-e^{-\beta \hbar \nu}} \int \frac{d \Omega}{2 \pi} \int \frac{d \omega}{2 \pi} \int \frac{d q}{2 \pi}\left[\tanh \left(\frac{\hbar(\Omega+v)}{2 k_{B} T}\right)-\tanh \left(\frac{\hbar \Omega}{2 k_{B} T}\right)\right] \\
& \times\left\{\operatorname{coth}\left(\frac{\hbar \Omega}{2 k_{B} T}\right)\left[d_{q}^{R}(-\omega)-d_{q}^{A}(-\omega)\right]\left(I^{+}(q)+I^{-}(q)-I^{+}(0)-I^{-}(0)\right)\right. \\
& -\tanh \left(\frac{\hbar(\Omega+v+\omega)}{2 k_{B} T}\right)\left[d_{q}^{R}(-\omega)\left(I^{+}(q)-I^{+}(0)\right)-d_{q}^{A}(-\omega)\left(I^{-}(q)-I^{-}(0)\right)\right] \\
& \left.-\tanh \left(\frac{\hbar(\Omega+\omega)}{2 k_{B} T}\right)\left[d_{q}^{R}(-\omega)\left(I^{-}(q)-I^{-}(0)\right)-d_{q}^{A}(-\omega)\left(I^{+}(q)-I^{+}(0)\right)\right]\right\} .
\end{aligned}
$$

We need to connect to Eq. (20), so we must obtain the momentum integrated susceptibility: $\chi^{(2)}(v)=(2 \pi)^{-2} \int d \boldsymbol{p} \chi^{(2)}(\boldsymbol{p}, v)$. Only the angular term $I^{ \pm}(q)$ carries external momentum, however, so the momentum integral can be carried out:

$$
\int_{0}^{\infty} \frac{p d p}{p^{2}} I^{ \pm}(q)=\int_{0}^{\infty} \frac{d x}{x} I^{ \pm}(q)=-\frac{\tan ^{-1}\left(q_{\perp} / k_{F s}\right)}{q / k_{F s}} .
$$

At this point, note that the integrand in Eq. (B22) is even in $q$, so it is possible to restrict the $q$ integral to half the domain. If we recall that there is an external sum over all lattice points, $\sum_{i}=N$, then because the quantum spin liquid is isotropic we have for the final result

$$
\chi^{(2)}(v)=\frac{1}{1-e^{-\beta \hbar v}} \frac{N m_{s}^{2} a_{s}^{2}}{2(2 \pi)^{2} \hbar^{4}} \int \frac{d \omega}{2 \pi} \int_{0}^{\infty} \frac{d q}{k_{F s}} F(v, \omega)\left[d_{q}^{R}(-\omega)-d_{q}^{A}(-\omega)\right]\left[1-\frac{\tan ^{-1}\left(q / k_{F s}\right)}{q / k_{F s}}\right],
$$

with

$$
F(v, \omega)=\int \frac{d \Omega}{2 \pi}\left[\tanh \left(\frac{\hbar(\Omega+v)}{2 k_{B} T}\right)-\tanh \left(\frac{\hbar \Omega}{2 k_{B} T}\right)\right]\left[2 \operatorname{coth}\left(\frac{\hbar \omega}{2 k_{B} T}\right)-\tanh \left(\frac{\hbar(\Omega+\omega+v)}{2 k_{B} T}\right)-\tanh \left(\frac{\hbar(\Omega+\omega)}{2 k_{B} T}\right)\right] .
$$

These are Eqs. (20) and (21) from the main text.

\section{APPENDIX C: THE KITAEV MODEL CALCULATION}

In this Appendix. we explicitly show our calculation of the retarded Green function for the Kitaev model calculation in Sec. IIIC. The unperturbed Hamiltonian for the $b$ fermions reads

$$
\mathcal{H}_{0}=\sum_{q}\left(\begin{array}{ll}
b_{\boldsymbol{q}}^{\dagger} & b_{-q}
\end{array}\right)\left(\begin{array}{cc}
\varepsilon_{\boldsymbol{q}} & -\Delta_{\boldsymbol{q}} \\
-\Delta_{q}^{*} & -\varepsilon_{\boldsymbol{q}}
\end{array}\right)\left(\begin{array}{c}
b_{\boldsymbol{q}} \\
b_{-q}^{\dagger}
\end{array}\right),
$$


where $\quad \varepsilon_{\boldsymbol{q}}=K\left(1+\cos \boldsymbol{q} \cdot \boldsymbol{n}_{1}+\cos \boldsymbol{q} \cdot \boldsymbol{n}_{2}\right), \quad \Delta_{\boldsymbol{q}}=$ $-\iota K\left(\sin \boldsymbol{q} \cdot \boldsymbol{n}_{1}+\sin \boldsymbol{q} \cdot \boldsymbol{n}_{2}\right)$, and $\boldsymbol{n}_{1}, \boldsymbol{n}_{2}$ are the real space lattice vectors as defined in the main text. The local potential across the $x$ bond of the $\boldsymbol{r}=0$ unit cell is given by

$$
V_{x}=u b_{0}^{\dagger} b_{0}-\frac{u}{2}=\frac{u}{N_{u}} \sum_{\boldsymbol{p q}} b_{p}^{\dagger} b_{\boldsymbol{q}}-\frac{u}{2},
$$

where $u=-4 K$ and $N_{u}$ is the number of unit cells in the lattice. Then the full Hamiltonian including the local potential is $H_{x}=\mathcal{H}_{0}+V_{x}$, for which we want to find the Green functions.

We take the equation of motion approach, so we require the commutation relations for $b_{k}$,

$$
\left[H_{x}, b_{k}\right]=-2 \varepsilon_{k} b_{k}+2 \Delta_{k} b_{-k}^{\dagger}-\frac{u}{N_{u}} \sum_{k} b_{k} .
$$

We now examine the retarded matrix Green functions in the Nambu basis, which we express as $G_{p q}^{R}(t, 0)=$ $\theta(t)\left[G_{p q}^{>}(t, 0)-G_{p q}^{<}(t, 0)\right]$ (note that capitalized $G$ Green functions will be reserved for matrices, and lower-case $g$ for components). The greater-than matrix Green functions is

$$
G_{p q}^{>}(t, 0)=-\iota\left(\begin{array}{cc}
\left\langle b_{p}(t) b_{-q}^{\dagger}(0)\right\rangle & \left\langle b_{p}(t) b_{q}(0)\right\rangle \\
\left\langle b_{-p}^{\dagger}(t) b_{-q}^{\dagger}(0)\right\rangle & \left\langle b_{-p}^{\dagger}(t) b_{q}(0)\right\rangle
\end{array}\right),
$$

and the lesser-than Green function matrix can be found by anticommuting the fermions in each component. Using Eq. (C3), we then find

$$
\begin{aligned}
\iota \partial_{t} G_{p q}^{R}(t, 0)= & \iota \delta(t) \delta_{p q}+\frac{2}{\hbar} H_{0}(\boldsymbol{p}) G_{p q}^{R}(t, 0) \\
& +\frac{u}{\hbar N} \sigma_{z} \sum_{p} G_{p q}^{R}(t, 0)
\end{aligned}
$$

where $H_{0}(\boldsymbol{p})$ is the coefficient matrix of $\mathcal{H}_{0}$, and $\sigma_{z}$ is the $z-$ component Pauli matrix. Fourier transforming to frequency

$$
G_{p q}^{R}(\omega)=G_{p}^{R, 0} \delta_{p q}+\frac{u}{\hbar N_{u}} G_{p}^{R, 0}(\omega) \sigma_{z} \sum_{k} G_{k q}^{R}(\omega),
$$

where the unperturbed Green function matrix for the $b_{k}$ fermions reads

$$
G_{p}^{R, 0}(\omega)=\frac{1}{\mathcal{N}_{\boldsymbol{p}}(\omega)}\left(\begin{array}{cc}
\omega+\frac{2}{\hbar} \varepsilon_{\boldsymbol{p}}+\iota \delta & \frac{2}{\hbar} \Delta_{\boldsymbol{p}} \\
\frac{2}{\hbar} \Delta_{\boldsymbol{p}}^{*} & \omega-\frac{2}{\hbar}+\iota \delta
\end{array}\right),
$$

and $\mathcal{N}_{p}(\omega)=(\omega+\iota \delta)^{2}-(2 / \hbar)^{2}\left|s_{p}\right|^{2}$. When summing over the momentum index, we can see that the off diagonal elements of $G_{p}^{R, 0}(\omega)$ vanish because $\Delta_{p}$ is an odd function of $\boldsymbol{p}$, and the Brillouin zone is symmetric about the origin. This is the reason there are no anomalous Green functions of the form $\left\langle b_{0}^{\dagger}(t) b_{0}^{\dagger}\left(t^{\prime}\right)\right\rangle$-or the complex conjugate-as mentioned in the main text. Thus we have $\sum_{p} G_{p}^{R, 0}(\omega)=$ $\operatorname{Diag}\left\{g_{11}^{R}(\omega), g_{22}^{R}(\omega)\right\}$, where the elements are

$$
g_{11,22}^{R}(\omega)=\sum_{\boldsymbol{p}} \frac{\omega \pm \frac{2}{\hbar} \varepsilon_{\boldsymbol{p}}+\iota \delta}{\mathcal{N}_{\boldsymbol{p}}(\omega)} .
$$

We then sum over $\boldsymbol{p}$ in Eq. (C6) in order to rewrite the full Green function in terms of unperturbed Green functions as

$$
\sum_{\boldsymbol{p}} G_{\boldsymbol{p} \boldsymbol{q}}^{R}(\omega)=\left[1-\frac{u}{\hbar N_{u}}\left(\sum_{\boldsymbol{p}} G_{\boldsymbol{p}}^{R, 0}(\omega)\right) \sigma_{z}\right]^{-1} G_{\boldsymbol{q}}^{R, 0}(\omega) .
$$

Finally, by substituting this expression back into Eq. (C6)

$$
\sum_{p \boldsymbol{q}} G_{\boldsymbol{p q}}^{R}(\omega)=\left(\begin{array}{cc}
\frac{g_{11}^{R}(\omega)}{1-\frac{u}{\hbar N_{u}} g_{11}^{R}(\omega)} & 0 \\
0 & \frac{g_{22}^{R}(\omega)}{1+\frac{u}{\hbar N_{u}} g_{22}^{R}(\omega)}
\end{array}\right) .
$$

The correlation function we require is $\left\langle b_{0}(t) b_{0}^{\dagger}(0)\right\rangle_{x}=$ $\left(1 / N_{u}\right) \sum_{p q}\left\langle b_{p}(t) b_{q}^{\dagger}(0)\right\rangle_{x}$, which in frequency space is $\iota g_{11}^{>}(\omega)$. In equilibrium at the quantum limit, $G^{>}(\omega)=$ $\theta(\omega)\left[G^{R}(\omega)-G^{A}(\omega)\right]$, and therefore we have

$$
g_{11}^{>}(\omega)=\theta(\omega)\left(\frac{g_{11}^{R}(\omega)}{1-\frac{u}{\hbar N_{u}} g_{11}^{R}(\omega)}-\frac{g_{11}^{A}(\omega)}{1-\frac{u}{\hbar N_{u}} g_{11}^{A}(\omega)}\right) .
$$

This is the expression used to compute the susceptibility in Eq. (28).
[1] L. Balents, Spin liquids in frustrated magnets, Nature (London) 464, 199 (2010).

[2] L. Savary and L. Balents, Quantum spin liquids: a review, Rep. Prog. Phys. 80, 016502 (2016).

[3] A. Kitaev and J. Preskill, Topological Entanglement Entropy, Phys. Rev. Lett. 96, 110404 (2006).

[4] M. Levin and X.-G. Wen, Detecting Topological Order in a Ground State Wave Function, Phys. Rev. Lett. 96, 110405 (2006).

[5] T. Senthil and M. P. A. Fisher, $Z_{2}$ gauge theory of electron fractionalization in strongly correlated systems, Phys. Rev. B 62, 7850 (2000).

[6] T. Senthil and M. P. A. Fisher, Fractionalization in the Cuprates: Detecting the Topological Order, Phys. Rev. Lett. 86, 292 (2001).

[7] M. R. Norman, Colloquium: Herbertsmithite and the search for the quantum spin liquid, Rev. Mod. Phys. 88, 041002 (2016).
[8] B. J. Powell and R. H. McKenzie, Quantum frustration in organic mott insulators: from spin liquids to unconventional superconductors, Rep. Prog. Phys. 74, 056501 (2011).

[9] H. Takagi, T. Takayama, G. Jackeli, G. Khaliullin, and Stephen E. Nagler, Concept and realization of kitaev quantum spin liquids, Nat. Rev. Phys. 1, 264 (2019).

[10] Y. Shimizu, K. Miyagawa, K. Kanoda, M. Maesato, and G. Saito, Spin Liquid State in an Organic Mott Insulator with a Triangular Lattice, Phys. Rev. Lett. 91, 107001 (2003).

[11] A. Olariu, P. Mendels, F. Bert, F. Duc, J. C. Trombe, M. A. de Vries, and A. Harrison, ${ }^{17} \mathrm{O} \mathrm{nmr}$ Study of the Intrinsic Magnetic Susceptibility and Spin Dynamics of the Quantum Kagome Antiferromagnet $\mathrm{ZnCu}_{3}(\mathrm{OH})_{6} \mathrm{Cl}_{2}$, Phys. Rev. Lett. 100, 087202 (2008).

[12] M. Fu, T. Imai, T.-H. Han, and Y. S. Lee, Evidence for a gapped spin-liquid ground state in a kagome Heisenberg antiferromagnet, Science 350, 655 (2015). 
[13] K. Kitagawa, T. Takayama, Y. Matsumoto, A. Kato, R. Takano, Y. Kishimoto, S. Bette, R. Dinnebier, G. Jackeli, and H. Takagi, A spin-orbital-entangled quantum liquid on a honeycomb lattice, Nature (London) 554, 341 (2018).

[14] F. Bert, S. Nakamae, F. Ladieu, D. L'Hôte, P. Bonville, F. Duc, J.-C. Trombe, and P. Mendels, Low temperature magnetization of the $s=\frac{1}{2}$ kagome antiferromagnet $\mathrm{ZnCu}_{3}(\mathrm{OH})_{6} \mathrm{Cl}_{2}$, Phys. Rev. B 76, 132411 (2007).

[15] M. Yamashita, N. Nakata, Y. Kasahara, T. Sasaki, N. Yoneyama, N. Kobayashi, S. Fujimoto, T. Shibauchi, and Y. Matsuda, Thermal-transport measurements in a quantum spin-liquid state of the frustrated triangular magnet $\kappa$-(bedt-ttf $)_{2} \mathrm{Cu}_{2}(\mathrm{CN})_{3}$, Nat. Phys. 5, 44 (2008).

[16] S. Yamashita, T. Yamamoto, Y. Nakazawa, M. Tamura, and R. Kato, Gapless spin liquid of an organic triangular compound evidenced by thermodynamic measurements, Nat. Commun. 2 , 275 (2011).

[17] S. Yamashita, Y. Nakazawa, M. Oguni, Y. Oshima, H. Nojiri, Y. Shimizu, K. Miyagawa, and K. Kanoda, Thermodynamic properties of a spin-1/2 spin-liquid state in a [kappa]-type organic salt, Nat. Phys. 4, 459 (2008).

[18] M. Yamashita, N. Nakata, Y. Senshu, M. Nagata, H. M. Yamamoto, R. Kato, T. Shibauchi, and Y. Matsuda, Highly mobile gapless excitations in a two-dimensional candidate quantum spin liquid, Science 328, 1246 (2010).

[19] D. Hirobe, M. Sato, Y. Shiomi, H. Tanaka, and E. Saitoh, Magnetic thermal conductivity far above the néel temperature in the kitaev-magnet candidate $\alpha-\mathrm{RuCl}_{3}$, Phys. Rev. B 95, 241112(R) (2017).

[20] Y. Kasahara, T. Ohnishi, Y. Mizukami, O. Tanaka, S. Ma, K. Sugii, N. Kurita, H. Tanaka, J. Nasu, Y. Motome, T. Shibauchi, and Y. Matsuda, Majorana quantization and half-integer thermal quantum hall effect in a kitaev spin liquid, Nature (London) 559, 227 (2018).

[21] P. Bourgeois-Hope, F. Laliberté, E. Lefrançois, G. Grissonnanche, S. R. de Cotret, R. Gordon, S. Kitou, H. Sawa, H. Cui, R. Kato, L. Taillefer, and N. Doiron-Leyraud, Thermal Conductivity of the Quantum Spin Liquid Candidate $\mathrm{EtMe}_{3} \mathrm{Sb}\left[\mathrm{Pd}(\mathrm{dmit})_{2}\right]_{2}$ : No Evidence of Mobile Gapless Excitations, Phys. Rev. X 9, 041051 (2019).

[22] J. M. Ni, B. L. Pan, Y. Y. Huang, J. Y. Zeng, Y. J. Yu, E. J. Cheng, L. S. Wang, R. Kato, and S. Y. Li, Absence of Magnetic Thermal Conductivity in the Quantum Spin Liquid Candidate $\mathrm{EtMe}_{3} \mathrm{Sb}\left[\mathrm{Pd}(\mathrm{dmit})_{2}\right]_{2}$, Phys. Rev. Lett. 123, 247204 (2019).

[23] J. S. Helton, K. Matan, M. P. Shores, E. A. Nytko, B. M. Bartlett, Y. Yoshida, Y. Takano, A. Suslov, Y. Qiu, J.-H. Chung, D. G. Nocera, and Y. S. Lee, Spin Dynamics of the Spin-1/2 Kagome Lattice Antiferromagnet $\mathrm{ZnCu}_{3}(\mathrm{OH})_{6} \mathrm{Cl}_{2}$, Phys. Rev. Lett. 98, 107204 (2007).

[24] M. A. de Vries, J. R. Stewart, P. P. Deen, J. O. Piatek, G. J. Nilsen, H. M. Rønnow, and A. Harrison, Scale-Free Antiferromagnetic Fluctuations in the $s=1 / 2$ Kagome Antiferromagnet Herbertsmithite, Phys. Rev. Lett. 103, 237201 (2009).

[25] J. S. Helton, K. Matan, M. P. Shores, E. A. Nytko, B. M. Bartlett, Y. Qiu, D. G. Nocera, and Y. S. Lee, Dynamic Scaling in the Susceptibility of the Spin- $\frac{1}{2}$ Kagome Lattice Antiferromagnet Herbertsmithite, Phys. Rev. Lett. 104, 147201 (2010).

[26] A. Banerjee, C. A. Bridges, J. Q. Yan, A. A. Aczel, L. Li, M. B. Stone, G. E. Granroth, M. D. Lumsden, Y. Yiu, J. Knolle, S. Bhattacharjee, D. L. Kovrizhin, R. Moessner, D. A. Tennant,
D. G. Mandrus, and S. E. Nagler, Proximate kitaev quantum spin liquid behavior in a honeycomb magnet, Nat. Mater. 15, 733 (2016).

[27] S. Sachdev, Kagomé- and triangular-lattice Heisenberg antiferromagnets: Ordering from quantum fluctuations and quantumdisordered ground states with unconfined bosonic spinons, Phys. Rev. B 45, 12377 (1992).

[28] T. Yildirim and A. B. Harris, Magnetic structure and spin waves in the kagomé jarosite compound $\mathrm{KFe}_{3}\left(\mathrm{SO}_{4}\right)_{2}(\mathrm{OH})_{6}$, Phys. Rev. B 73, 214446 (2006).

[29] S. Yan, D. A. Huse, and S. R. White, Spin-liquid ground state of the $\mathrm{s}=1 / 2$ kagome Heisenberg antiferromagnet, Science 332, 1173 (2011).

[30] H. O. Jeschke, F. Salvat-Pujol, and R. Valentí, First-principles determination of Heisenberg Hamiltonian parameters for the spin- $\frac{1}{2}$ kagome antiferromagnet $\mathrm{ZnCu}_{3}(\mathrm{OH})_{6} \mathrm{Cl}_{2}$, Phys. Rev. B 88, 075106 (2013).

[31] Y.-C. He, M. P. Zaletel, M. Oshikawa, and F. Pollmann, Signatures of Dirac Cones in a Dmrg Study of the Kagome Heisenberg Model, Phys. Rev. X 7, 031020 (2017).

[32] O. I. Motrunich, Variational study of triangular lattice spin- $\frac{1}{2}$ model with ring exchanges and spin liquid state in $\kappa-(\mathrm{ET})_{2} \mathrm{Cu}_{2}(\mathrm{CN})_{3}$, Phys. Rev. B 72, 045105 (2005).

[33] S.-S. Lee and P. A. Lee, U(1) Gauge Theory of the Hubbard Model: Spin Liquid States and Possible Application to $\kappa$-(BEDT-TTF $)_{2} \mathrm{Cu}_{2}(\mathrm{CN})_{3}$, Phys. Rev. Lett. 95, 036403 (2005).

[34] C. P. Nave and P. A. Lee, Transport properties of a spinon fermi surface coupled to a U(1) gauge field, Phys. Rev. B 76, 235124 (2007).

[35] S.-S. Lee, Stability of the U(1) spin liquid with a spinon fermi surface in $2+1$ dimensions, Phys. Rev. B 78, 085129 (2008).

[36] S.-S. Lee, Low-energy effective theory of fermi surface coupled with U(1) gauge field in $2+1$ dimensions, Phys. Rev. B 80, 165102 (2009).

[37] S. Sanyal, K. Dhochak, and S. Bhattacharjee, Interplay of uniform $u(1)$ quantum spin liquid and magnetic phases in rare-earth pyrochlore magnets: A fermionic parton approach, Phys. Rev. B 99, 134425 (2019).

[38] A. Kitaev, Anyons in an exactly solved model and beyond, Ann. Phys. 321, 2 (2006).

[39] G. Jackeli and G. Khaliullin, Mott Insulators in the Strong SpinOrbit Coupling Limit: From Heisenberg to a Quantum Compass and Kitaev Models, Phys. Rev. Lett. 102, 017205 (2009).

[40] J. Knolle, D. L. Kovrizhin, J. T. Chalker, and R. Moessner, Dynamics of a Two-Dimensional Quantum Spin Liquid: Signatures of Emergent Majorana Fermions and Fluxes, Phys. Rev. Lett. 112, 207203 (2014).

[41] S. Mandal and N. Surendran, Exactly solvable kitaev model in three dimensions, Phys. Rev. B 79, 024426 (2009).

[42] J. Nasu, M. Udagawa, and Y. Motome, Vaporization of Kitaev Spin Liquids, Phys. Rev. Lett. 113, 197205 (2014).

[43] J. Knolle and R. Moessner, A field guide to spin liquids, Annu. Rev. Condens. Matter Phys. 10, 451 (2019).

[44] E. Saitoh, M. Ueda, H. Miyajima, and G. Tatara, Conversion of spin current into charge current at room temperature: Inverse spin-hall effect, Appl. Phys. Lett. 88, 182509 (2006).

[45] J. Sinova, S. O. Valenzuela, J. Wunderlich, C. H. Back, and T. Jungwirth, Spin Hall effects, Rev. Mod. Phys. 87, 1213 (2015). 
[46] K. Uchida, S. Takahashi, K. Harii, J. Ieda, W. Koshibae, K. Ando, S. Maekawa, and E. Saitoh, Observation of the spin seebeck effect, Nature (London) 455, 778 (2008).

[47] K. Uchida, H. Adachi, T. Ota, H. Nakayama, S. Maekawa, and E. Saitoh, Observation of longitudinal spin-seebeck effect in magnetic insulators, Appl. Phys. Lett. 97, 172505 (2010).

[48] D. Hirobe, M. Sato, T. Kawamata, Y. Shiomi, Ken-ichi Uchida, R. Iguchi, Y. Koike, S. Maekawa, and E. Saitoh, Onedimensional spinon spin currents, Nat. Phys. 13, 30 (2017).

[49] Y. Kajiwara, K. Harii, S. Takahashi, J. Ohe, K. Uchida, M. Mizuguchi, H. Umezawa, H. Kawai, K. Ando, K. Takanashi, S. Maekawa, and E. Saitoh, Transmission of electrical signals by spin-wave interconversion in a magnetic insulator, Nature (London) 464, 262 (2010).

[50] L. J. Cornelissen, J. Liu, R. A. Duine, J. Ben Youssef, and B. J. van Wees, Long-distance transport of magnon spin information in a magnetic insulator at room temperature, Nat. Phys. 11, 1022 (2015).

[51] S. T. B. Goennenwein, R. Schlitz, M. Pernpeintner, K. Ganzhorn, M. Althammer, R. Gross, and H. Huebl, Non-local magnetoresistance in YIG/Pt nanostructures, Appl. Phys. Lett. 107, 172405 (2015).

[52] J. Li, Y. Xu, M. Aldosary, C. Tang, Z. Lin, S. Zhang, R. Lake, and J. Shi, Observation of magnon-mediated current drag in $\mathrm{Pt} / \mathrm{yttrium}$ iron garnet/Pt(Ta) trilayers, Nat. Commun. 7, 10858 (2016).

[53] W. Han, S. Maekawa, and X.-C. Xie, Spin current as a probe of quantum materials, Nat. Mater. 19, 139 (2019).

[54] H. B. Callen and T. A. Welton, Irreversibility and generalized noise, Phys. Rev. 83, 34 (1951).

[55] R. Kubo, The fluctuation-dissipation theorem, Rep. Prog. Phys. 29, 255 (1966).

[56] A. Kamra and W. Belzig, Magnon-mediated spin current noise in ferromagnet | nonmagnetic conductor hybrids, Phys. Rev. B 94, 014419 (2016).

[57] A. A. Clerk, M. H. Devoret, S. M. Girvin, F. Marquardt, and R. J. Schoelkopf, Introduction to quantum noise, measurement, and amplification, Rev. Mod. Phys. 82, 1155 (2010).

[58] S. Florens and A. Georges, Slave-rotor mean-field theories of strongly correlated systems and the mott transition in finite dimensions, Phys. Rev. B 70, 035114 (2004).

[59] J. Nasu, M. Udagawa, and Y. Motome, Thermal fractionalization of quantum spins in a kitaev model: Temperature-linear specific heat and coherent transport of majorana fermions, Phys. Rev. B 92, 115122 (2015).

[60] I. Rousochatzakis, S. Kourtis, J. Knolle, R. Moessner, and N. B. Perkins, Quantum spin liquid at finite temperature: Proximate dynamics and persistent typicality, Phys. Rev. B 100, 045117 (2019).

[61] L. A. Hall, Survey of Electrical Resistivity Measurements on 16 Pure Metals in the Temperature Range 0 to $273^{\circ} \mathrm{K}$, National Bureau of Standards, Boulder, CO (1968).

[62] P. D. Desai, T. K. Chu, H. M. James, and C. Y. Ho, Electrical resistivity of selected elements, J. Phys. Chem. Ref. Data 13, 1069 (1984).

[63] D. G. Joshi, A. P. Schnyder, and S. Takei, Detecting end states of topological quantum paramagnets via spin hall noise spectroscopy, Phys. Rev. B 98, 064401 (2018).
[64] A. Kamra, F. P. Witek, S. Meyer, H. Huebl, S. Geprägs, R. Gross, G. E. W. Bauer, and S. T. B. Goennenwein, Spin Hall noise, Phys. Rev. B 90, 214419 (2014).

[65] F. Wang and A. Vishwanath, Spin-liquid states on the triangular and kagomé lattices: A projective-symmetry-group analysis of Schwinger boson states, Phys. Rev. B 74, 174423 (2006).

[66] M. Punk, D. Chowdhury, and S. Sachdev, Topological excitations and the dynamic structure factor of spin liquids on the kagome lattice, Nat. Phys. 10, 289 (2014).

[67] Y.-M. Lu, Y. Ran, and P. A. Lee, $\mathbb{Z}_{2}$ spin liquids in the $s=\frac{1}{2}$ Heisenberg model on the kagome lattice: A projective symmetry-group study of schwinger fermion mean-field states, Phys. Rev. B 83, 224413 (2011).

[68] Y. Huh, L. Fritz, and S. Sachdev, Quantum criticality of the kagome antiferromagnet with Dzyaloshinskii-Moriya interactions, Phys. Rev. B 81, 144432 (2010).

[69] S. Chatterjee and S. Sachdev, Probing excitations in insulators via injection of spin currents, Phys. Rev. B 92, 165113 (2015).

[70] Y.-D. Li, X. Wang, and G. Chen, Anisotropic spin model of strong spin-orbit-coupled triangular antiferromagnets, Phys. Rev. B 94, 035107 (2016).

[71] C. Liu, R. Yu, and X. Wang, Semiclassical ground-state phase diagram and multi- $q$ phase of a spin-orbit-coupled model on triangular lattice, Phys. Rev. B 94, 174424 (2016).

[72] Y.-D. Li, Y.-M. Lu, and G. Chen, Spinon fermi surface U(1) spin liquid in the spin-orbit-coupled triangular-lattice mott insulator $\mathrm{YbMgGaO}_{4}$, Phys. Rev. B 96, 054445 (2017).

[73] P. A. Lee and N. Nagaosa, Gauge theory of the normal state of high- $t_{c}$ superconductors, Phys. Rev. B 46, 5621 (1992).

[74] J. Polchinski, Low-energy dynamics of the spinon-gauge system, Nucl. Phys. B 422, 617 (1994).

[75] Y. B. Kim, A. Furusaki, X.-G. Wen, and P. A. Lee, Gaugeinvariant response functions of fermions coupled to a gauge field, Phys. Rev. B 50, 17917 (1994).

[76] L. Balents and O. A. Starykh, Spinon waves in magnetized spin liquids, Phys. Rev. B 101, 020401 (2020).

[77] J. Knolle, D. L. Kovrizhin, J. T. Chalker, and R. Moessner, Dynamics of fractionalization in quantum spin liquids, Phys. Rev. B 92, 115127 (2015).

[78] M. Hermanns, I. Kimchi, and J. Knolle, Physics of the kitaev model: Fractionalization, dynamic correlations, and material connections, Annu. Rev. Condens. Matter Phys. 9, 17 (2018).

[79] J. Knolle, S. Bhattacharjee, and R. Moessner, Dynamics of a quantum spin liquid beyond integrability: The kitaevHeisenberg- $\Gamma$ model in an augmented parton mean-field theory, Phys. Rev. B 97, 134432 (2018).

[80] E. H. Lieb, Flux Phase of the Half-Filled Band, Phys. Rev. Lett. 73, 2158 (1994).

[81] G. Baskaran, S. Mandal, and R. Shankar, Exact Results for Spin Dynamics and Fractionalization in the Kitaev Model, Phys. Rev. Lett. 98, 247201 (2007).

[82] S.O. Kasap, Principles of Electronic Materials and Devices, McGraw-Hill International Edition (McGraw-Hill, New York, 2006).

[83] T.-H. Han, J. S. Helton, S. Chu, D. G. Nocera, J. A. RodriguezRivera, C. Broholm, and Y. S. Lee, Fractionalized excitations in the spin-liquid state of a kagome-lattice antiferromagnet, Nature (London) 492, 406 (2012). 
[84] T.-H. Han, M. R. Norman, J.-J. Wen, J. A. RodriguezRivera, J. S. Helton, C. Broholm, and Y. S. Lee, Correlated impurities and intrinsic spin-liquid physics in the kagome material herbertsmithite, Phys. Rev. B 94, 060409(R) (2016).

[85] D. E. Freedman, T. H. Han, A. Prodi, P. Müller, Q.Z. Huang, Y.-S. Chen, S. M. Webb, Y. S. Lee, T. M. McQueen, and D. G. Nocera, Site specific X-ray anomalous dispersion of the geometrically frustrated kagomémagnet, herbertsmithite, $\mathrm{ZnCu}_{3}(\mathrm{OH})_{6} \mathrm{Cl}_{2}$, J. Am. Chem. Soc. 132, 16185 (2010).

[86] I. Kimchi, A. Nahum, and T. Senthil, Valence Bonds in Random Quantum Magnets: Theory and Application to $\mathrm{YbMgGaO}_{4}$, Phys. Rev. X 8, 031028 (2018).

[87] L. Ding, P. Manuel, S. Bachus, F. Grußler, P. Gegenwart, J. Singleton, R. D. Johnson, H. C. Walker, D. T. Adroja, A. D. Hillier, and A. A. Tsirlin, Gapless spin-liquid state in the structurally disorder-free triangular antiferromagnet $\mathrm{NaYbO}_{2}$, Phys. Rev. B 100, 144432 (2019).

[88] J. Chaloupka, G. Jackeli, and G. Khaliullin, Zigzag Magnetic Order in the Iridium Oxide $\mathrm{Na}_{2} \mathrm{IrO}_{3}$, Phys. Rev. Lett. 110, 097204 (2013).

[89] K. W. Plumb, J. P. Clancy, L. J. Sandilands, V. V. Shankar, Y. F. $\mathrm{Hu}, \mathrm{K} . \mathrm{S}$. Burch, H.-Y. Kee, and Y.-J. Kim, $\alpha-\mathrm{RuCl}_{3}$ : A spinorbit assisted mott insulator on a honeycomb lattice, Phys. Rev. B 90, 041112(R) (2014).

[90] X. Liu, T. Berlijn, W.-G. Yin, W. Ku, A. Tsvelik, Y.-J. Kim, H. Gretarsson, Y. Singh, P. Gegenwart, and J. P. Hill, Long-range magnetic ordering in $\mathrm{Na}_{2} \mathrm{IrO}_{3}$, Phys. Rev. B 83, 220403(R) (2011).

[91] S. K. Choi, R. Coldea, A. N. Kolmogorov, T. Lancaster, I. I. Mazin, S. J. Blundell, P. G. Radaelli, Y. Singh, P. Gegenwart, K. R. Choi, S.-W. Cheong, P. J. Baker, C. Stock, and J. Taylor,
Spin Waves and Revised Crystal Structure of Honeycomb Iridate $\mathrm{Na}_{2} \mathrm{IrO}_{3}$, Phys. Rev. Lett. 108, 127204 (2012).

[92] Y. Kubota, H. Tanaka, T. Ono, Y. Narumi, and K. Kindo, Successive magnetic phase transitions in $\alpha-\mathrm{RuCl}_{3}$ : Xy-like frustrated magnet on the honeycomb lattice, Phys. Rev. B 91, 094422 (2015).

[93] S. Hwan Chun, J.-W. Kim, J. Kim, H. Zheng, C. C. Stoumpos, C. D. Malliakas, J. F. Mitchell, K. Mehlawat, Y. Singh, Y. Choi, T. Gog, A. Al-Zein, M. M. Sala, M. Krisch, J. Chaloupka, G. Jackeli, G. Khaliullin, and B. J. Kim, Direct evidence for dominant bond-directional interactions in a honeycomb lattice iridate $\mathrm{Na}_{2} \mathrm{IrO}_{3}$, Nat. Phys. 11, 462 (2015).

[94] M. Majumder, M. Schmidt, H. Rosner, A. A. Tsirlin, H. Yasuoka, and M. Baenitz, Anisotropic $\mathrm{Ru}^{3+} 4 d^{5}$ magnetism in the $\alpha-\mathrm{RuCl}_{3}$ honeycomb system: Susceptibility, specific heat, and zero-field NMR, Phys. Rev. B 91, 180401(R) (2015).

[95] L. J. Sandilands, Y. Tian, K. W. Plumb, Y.-J. Kim, and K. S. Burch, Scattering Continuum and Possible Fractionalized Excitations in $\alpha-\mathrm{RuCl}_{3}$, Phys. Rev. Lett. 114, 147201 (2015).

[96] S. M. Winter, K. Riedl, P. A. Maksimov, A. L. Chernyshev, A. Honecker, and R. Valentí, Breakdown of magnons in a strongly spin-orbital coupled magnet, Nat. Commun. 8, 1152 (2017).

[97] E. Onac, F. Balestro, L. H. W. van Beveren, U. Hartmann, Y. V. Nazarov, and L. P. Kouwenhoven, Using a Quantum dot as a High-Frequency Shot Noise Detector, Phys. Rev. Lett. 96, 176601 (2006).

[98] Y. Niimi, Y. Kawanishi, D. H. Wei, C. Deranlot, H. X. Yang, M. Chshiev, T. Valet, A. Fert, and Y. Otani, Giant Spin Hall Effect Induced by Skew Scattering from Bismuth Impurities Inside Thin Film CuBi Alloys, Phys. Rev. Lett. 109, 156602 (2012).

[99] A. Kamenev, Field Theory of Non-Equilibrium Systems (Cambridge University Press, Cambridge, 2011). 